\title{
Global software development governance: Challenges and solutions
}

\section{Antonio Manjavacas 이 | Aurora Vizcaíno 주 | Francisco Ruiz | Mario Piattini}

University of Castilla-La Mancha, Ciudad Real, Spain

Correspondence

Aurora Vizcaíno, University of Castilla-La

Mancha, Paseo de la Universidad, 4, 13071

Ciudad Real, Spain.

Email: aurora.vizcaino@uclm.es

Funding information

BIZDEVOPS-GLOBAL project; G3SOFT project; Ministerio de Ciencia, Innovación y Universidades, y Fondo Europeo de Desarrollo Regional FEDER, Grant/Award Number: RTI2018-098309-B-C31; Consejería de Educación, Cultura y Deportes de la Junta de Comunidades de Castilla La Mancha, y Fondo Europeo de Desarrollo Regional FEDER, Grant/ Award Number: SBPLY/17/180501/000150

\begin{abstract}
Global software development (GSD) has become a rising software development model in the last few years. Although much research has been performed in terms of GSD management, GSD governance research is scarce at the present time and presents multiple challenges that need to be addressed. In this paper, a systematic mapping study has been conducted, the aim of which was to discover the main issues dealt with in GSD governance literature, as well as to point out the particular research gaps that are still present in this domain. This allowed us to find out new challenges to be addressed, along with possible solutions to these. The results reveal a lack of research in key aspects such as tools, culture, people, and information, all of which provides a great opportunity for future approaches while also highlighting new opportunities in GSD governance research.
\end{abstract}

\section{KEYWORDS}

global software development, governance, software engineering, systematic mapping study

\section{1 | INTRODUCTION}

The decentralized development model known as global software development (GSD) has become a settled industrial software production model over the last decade. The main benefit that GSD brings to enterprises is that it provides access to talent and the market and that it offers flexibility according to business needs, thereby reducing time to market and production costs. ${ }^{1}$ The trend towards outsourcing software development is based on the search for benefits associated with resource pools, attractive cost structures, and round-the-clock development. ${ }^{2}$ There are other "unknown" advantages such as innovation, shared best practices, improved documentation, clearly defined processes, or increased team autonomy. ${ }^{3,4}$

However, despite the multiple benefits that GSD has brought to the software industry, there are still challenges that need to be addressed. Many of them have been tackled at the same time as GSD was being consolidated as a business model; they include such aspects as geographical, temporal, and sociocultural distances, all of which have been a key challenge in GSD research from its beginnings. ${ }^{5,6}$ The influence of these distances on collaborative communication undermines project outcomes and performance, as well as the relationships between stakeholders. ${ }^{7,8}$ Other barriers found in the literature have been lack of trust between individuals, which reduces the frequency of communication activities, ${ }^{9}$ and software architecting issues. ${ }^{10}$ All of the above determine, among other things, the work allocation between distributed teams. The introduction of agile practices, with the consequent challenges that this has entailed, has been another issue especially addressed in GSD research over the last few years. ${ }^{10-12}$

Faced with all these challenges, multiple solutions have been proposed from a managerial perspective: frameworks, theoretical models, tools, and case studies, where many good practices have been recommended. Some trends are shown in Ebert et al. ${ }^{13}$ where collaboration between teams, processes, organization sourcing, supplier management, and success factors stands out as the most relevant topics in GSD research. But are there as many proposed solutions from a governance point of view? Our experience in this field has made us aware of the fact that, although 
many challenges present in GSD management are being addressed, there are still many research gaps in the area of GSD governance that also need to be tackled.

Governance has a fundamental role in every IT business nowadays. The process of making strategic decisions, adapted to the situation of a given business model and aimed at the optimal use of resources, is a responsibility that falls on governance; it also directly affects the management of the organization, hence its importance.

As described in Bannerman, ${ }^{14}$ governance involves the set of arrangements and practices that an organization puts in place to ensure proper management. This "managing the management of the organization" is defined by him as meta-management. More specifically, IT governance has the responsibility of ensuring that the results of the business processes are in line with the strategic requirements defined in the organization. ${ }^{15}$ At the same time, these processes must be governed, if the reliability and efficiency of the software on which they depend is to be ensured. ${ }^{16}$

Governance is even more critical in distributed environments, where lack of awareness among offshore teams, sociocultural differences, and limited resources, determine key factors such as proper communication, clear decision-making, task allocation, and goal alignment. ${ }^{17}$ This idea is supported by Dubinsky et al. ${ }^{18}$ where it is explained that GSD governance is of particular relevance, due to software organizations' need to operate globally in complex and turbulent environments.

As it can be observed, given the relevance of GSD governance and the scarcity of research in this field when compared with that of management, knowing the state of the art in GSD governance is certainly an objective to be achieved. In an effort to learn what challenges have been addressed and which ones are still pending in GSD governance research and application, we, therefore, decided to carry out an exhaustive study of the literature in this area.

A starting point when defining how governance objectives should be achieved in practice is COBIT (Control Objectives for Information and Related Technologies). ${ }^{19}$ Held by ISACA, this framework has been established as a de facto industry standard in researching, developing, establishing, and promoting control objectives focused on IT governance and management. COBIT 2019 defines components, design factors, and governance problems that must be managed in the quest to guarantee the assurance of the stakeholders' needs, correct decision-making and business orientation, and the monitoring and improvement of the management processes. ${ }^{19} \mathrm{COBIT}$ also provides a definition of the main governance duties, which must ensure that

- Stakeholders' needs, conditions, and options are considered, assessed, and aligned with business objectives.

- Direction is set through prioritization and decision-making.

- Performance and compliance are monitored according to direction and objectives.

On this basis, in an effort to discover the state of the art as regards GSD governance, we decided to use COBIT 2019 as a means to systematically map all the publications in literature that are related to this topic. We first classified the research works concerning GSD governance according to the objectives defined in COBIT 2019, ${ }^{20}$ before going on to identify the COBIT components addressed in each of these publications. This enabled us to acquire a better understanding of the current situation of GSD governance, in addition to highlighting research gaps in this field, revealing new challenges and showing possible solutions to be addressed. No study of these characteristics has, to the best of our knowledge, been carried out in this domain to date.

With regard to the domain we are dealing with in our research, although there is a broad consensus on the fusion of local and distributed governance from the GSD academic community, the main interest of this work lies in the concrete understanding of problems and solutions that concern only decentralized software engineering. We, therefore, believe that the main contribution of this study is that of providing an overview of the governance objectives in GSD and how these objectives have been addressed over time in literature. The use of COBIT as a guiding framework may in turn help practitioners to identify unique challenges to these environments, beyond local governance duties.

Having introduced the purpose of this paper, in Section 2, other mappings and systematic literature reviews on GSD governance and management will be mentioned. In Section 3, the literature study and mapping procedure performed will be presented, along with the results obtained for each category. Section 4 will be used to address challenges and open issues in the GSD governance domain. Finally, validity threats will be commented on and the main conclusions of this work will be drawn in Sections 5 and 6, respectively.

\section{2 | RELATED WORK}

Many systematic reviews have been conducted within the scope of GSD. Some of the most recent systematic literature reviews and mapping studies in this domain have identified solutions, challenges or research gaps in terms of software architecting, ${ }^{10} \mathrm{knowledge} \mathrm{sharing,}^{21-23}$ agile practices, ${ }^{12,24}$ requirements risks and change management, ${ }^{25-29}$ cultural differences ${ }^{28,29}$ or process improvement, ${ }^{30}$ and among others. Although some of these studies address aspects that could indirectly be related to GSD governance, none of them directly addresses governance issues. 
We can also find some systematic reviews that have been conducted as regards IT governance, such as the work in Alves et al. ${ }^{31}$ where software ecosystems are studied in detail, identifying governance mechanisms suitable to this kind of environments. On the other hand, in Luna et al. $^{32}$ the current challenge of research in agile governance is addressed, highlighting the need for researchers and practitioners to work together to establish reciprocal research agenda in this area. Nevertheless, none of the existing reviews about IT governance deals with the GSD issues.

It is a well-known fact that systematic mapping studies and literature reviews on GSD governance are scarce, as explained in Noll et al. ${ }^{17}$ where research in software development governance is defined as being less mature and models for GSD governance are said to be missing.

As we have seen, many aspects have been addressed in dealing with GSD, but governance still seems to be disregarded. To the best of our knowledge, there is as yet no systematic literature review or systematic mapping of GSD governance literature. This explains our interest in knowing what solutions have been addressed so far, as well as in ascertaining what research gaps and challenges remain to be resolved in this domain.

\section{3 | RESEARCH PROCEDURE}

Being aware of the lack of publications on IT governance in GSD settings, we decided to carry out a systematic mapping study in this field and opted to use COBIT as a means of discovering which governance objectives and components stated in this framework have been addressed most frequently in literature. The basis employed for this purpose was principally the procedures set out in Kitchenham et al. ${ }^{33}$ which were taken as a reference for this study. We additionally employed the works of Budgen et al. ${ }^{34}$ and Petersen et al. ${ }^{35}$ as complements in order to outline the design of this research.

On this basis, the following subsections will provide a step-by-step description of the systematic mapping study process performed.

\section{1 | Research questions}

The following research questions were considered as needing to be addressed in our systematic mapping study:

RQ1: Which COBIT objectives have been addressed in GSD governance research?

RQ2: Which COBIT components have been addressed in GSD governance research?

On the one hand, RQ1 will allow us to discover which areas of GSD governance have been treated most in the literature, it will also enable us to identify research gaps in the field; at the same time, it will point to future challenges that need to be addressed. On the other hand, RQ2 will allow us to know which types of solutions are most commonly proposed for each of the objectives in the literature, as well as to identify which ones have not yet been dealt with sufficiently.

\subsection{Search procedure}

The search process set out in Figure 1 consisted of a manual search in the Scopus and ACM Digital Library databases. As the IEEE Digital Library content is currently indexed in Scopus, ${ }^{\dagger}$ the publication search was reduced to only two sources.

Although the specific search strings for each of the databases are detailed in Appendix B, the generic search string used was the following:

("governance" AND ("global software development" OR "distributed software development" OR "global software engineering" OR "gsd" OR "gse" OR "distributed software development" OR "distributed software engineering" OR "multi-site software development" OR "multi-site software engineering") AND "software")

The use of the term "software" at the end of the string may seem redundant, but it was included in an effort to avoid papers that included the acronym "gsd" (global software development) or "gse" (global software engineering) and that did not address software engineering topics. This allowed a large number of publications to be discarded directly during the search process.

The first search process was carried out on 7 April 2019 for all databases, so only papers published up to this date were included. Moreover, the date of completion of the study was December 2019.

thttps://supportcenter.ieee.org/app/answers/detail/a_id/510/ /is-ieee-xplore-digital-library-content-indexed-in-scopus\%3F. 


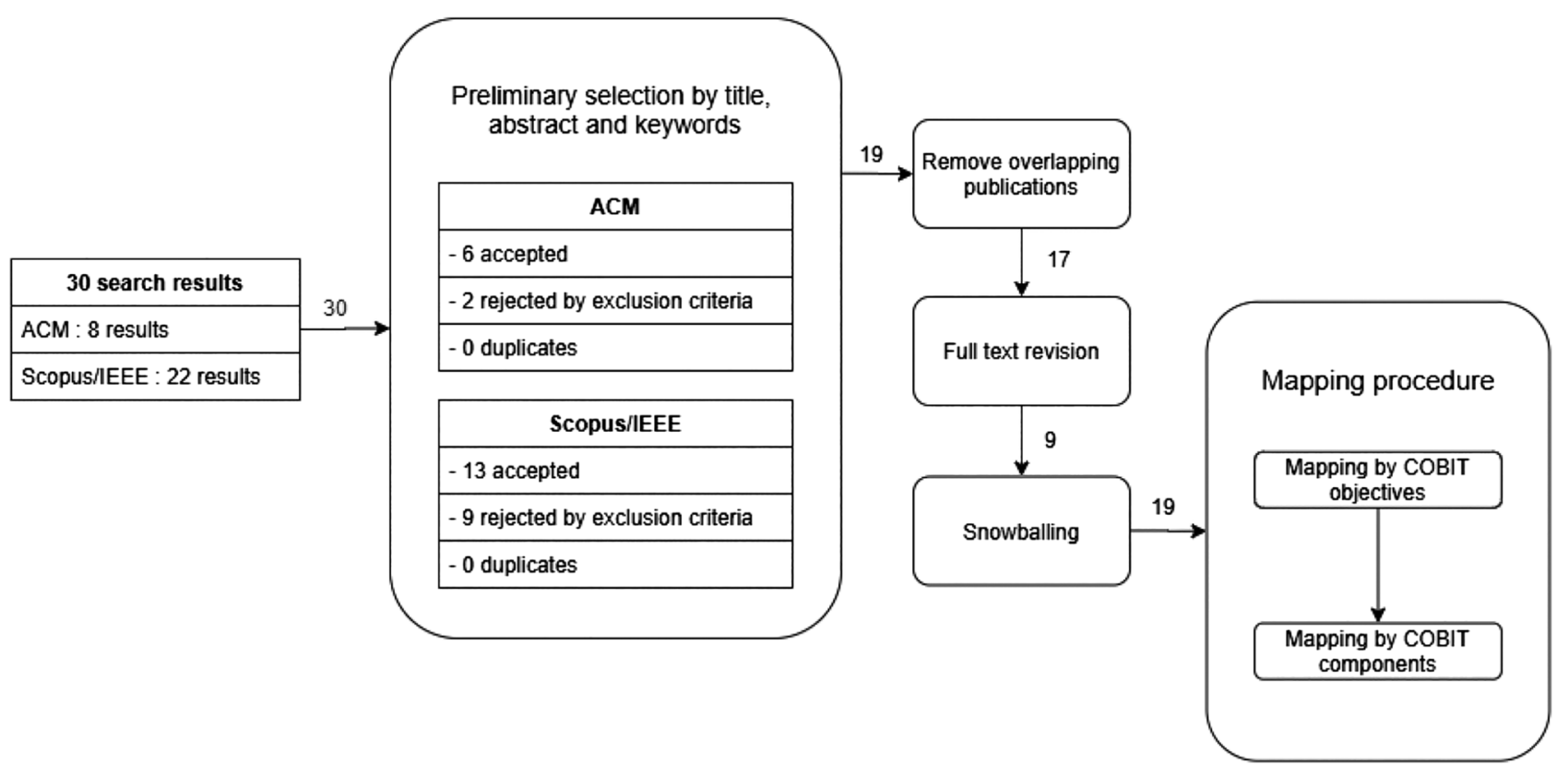

FIGURE 1 Search, filtering, and mapping procedure

\subsection{Inclusion and exclusion criteria}

The following criteria were considered during the selection of pertinent publications for our study:

a Inclusion criteria

- Publications concerning governance in GSD that

- offer applicable solutions (tools, frameworks, processes, etc.) in the field of this domain,

- present experiences or case studies directly related to the duties and competences of GSD governance, or

- address theoretical studies in this area, as systematic literature reviews and systematic mappings studies of the literature in question.

b Exclusion criteria

- Publications prior to 2000 , as global software development was not consolidated until the early years of that decade, with the first edition of ICGSE being in 2006;

- works published after April 2019, when the first search for the papers presented in this study was performed;

- duplicated reports of the same study: similar papers obtained from different sources, with the same title and authors;

- papers that are not written in English;

- conference proceedings that only list papers;

- publications that address GSD in academic settings only, such as in-class or teaching experiences, which are not suitable for practitioners; and

- search string results that are not related to the field of software engineering in GSD.

\section{4 | Quality assessments}

The following quality criteria ${ }^{33}$ were taken into account when considering an objective choice of the publications to be mapped. These were chosen by the authors in a consensual way and served to accept or rule out those publications that distanced themselves from the objective of our study:

QA1: Are the objectives of the paper clearly stated, defined and addressed?

- Yes (+1): the objectives are clearly stated and rigorously addressed.

- Partly (+0.5): the stated objectives are covered without extensive detail or are not clearly expressed. 
- No (+0): no specific objectives are specified or the objectives addressed are ambiguous.

- Unknown.

QA2: Are the results of the paper discussed rather than merely of just presented?

- Yes (+1): a critical and valuable perspective of the results obtained is provided.

- Partly (+0.5): a brief reflection on the results takes place without going into great details.

- No (+0): the results are presented without being judged.

- Unknown.

QA3: Does the paper clearly address topics that are closely bound to the COBIT objectives by which they could be classified?

- Yes (+1): the topic of the paper is closely related to a COBIT objective.

- Partly (+0.5): the paper indirectly addresses a COBIT objective.

No (+0): the topic of the paper is not related to any COBIT objective.

- Unknown.

QA4: Does the paper clearly address topics that are closely bound to the COBIT components by which they could be classified?

- Yes (+1): the topic of the paper is closely related to some COBIT component.

- Partly (+0.5): the paper indirectly addresses some COBIT component.

No (+0): the topic of the paper is not related to any COBIT component.

Unknown.

QA5: Does the publication properly describe tools, frameworks, or methods designed or used within the scope of its related COBIT objective?

- Yes (+1): the paper clearly describes proved tools, frameworks or methods employed to address challenges in GSD governance.

- Partly (+0.5): the paper describes unproved tools, frameworks or methods employed to address challenges in GSD governance.

- No (+0): the paper does not describe tools, frameworks or methods employed to address challenges in GSD governance.

- Unknown.

For any publication to be considered as a relevant work, it was decided that the final quality score should be higher than 3.

Thus, whenever there were disagreements between the authors, a joint discussion about the decisions would take place until an agreement was reached. The same procedure was carried out when there was any unknown appraisal. This process was carried out iteratively for each of the discrepancies, producing the results that we will set out in detail in later sections. A summary of the results can be found in Appendix D.

\section{5 | Data extraction}

Once all the publications obtained using the search string had been compiled, the StArt tool ${ }^{36}$ was employed in order to conduct a staggered selection and filtering process of the publications. After exporting the results of the search string in each source to the tool, the following of paper extraction process was performed:

- First, a selection by title, keywords, and abstract was undertaken, following the inclusion and exclusion criteria already mentioned. A total of 11 papers were discarded after this process, because they did not conform to the subject matter of our research. Of the remaining 19 papers, two overlapping publications found in both search sources were removed, signifying that we eventually attained 17 papers focused on the subject matter.

- The papers that preliminarily met the selection criteria were then assessed by being read in full by employing the abovementioned quality assessments. The fulfillment of these quality objectives would imply a correct adjustment to the subject matter of the research. An essential aspect pursued was that the papers extracted at this stage should reflect research into some kind of COBIT governance objective, either by defining challenges or solutions concerning these objectives. Of the 17 papers obtained prior to this phase, nine met the defined requirements.

- Once we had collected all the papers that had passed the selection and quality filters, the snowballing procedure ${ }^{37}$ was performed. This was done with the intention of seeking additional papers referenced in those that had been completely reviewed. The papers attained after snowballing were obtained by carrying out an iterative search based on the references of the initial papers. The publications eventually considered were therefore, for the most part, publications in which GSD governance is dealt with indirectly or in which this subject is addressed but could not be located by merely studying the title, abstract, and keywords. These papers were assessed according to the same quality criteria as the other publications and were either discarded or accepted on the basis of these criteria. A total of 10 papers were obtained after this process and were added to those already compiled.

- Finally, all the studies were transposed onto a spreadsheet, on which the mapping by objectives and components process was undertaken. A series of readings of each of the papers was then carried out in an attempt to identify the COBIT objectives covered, in addition to providing a justification for each mapping step. 
The first mapping step was the classification of the papers according to the governance objectives defined in COBIT 2019, followed by the classification according to governance components. Several iterations were necessary to reach an agreement on each of the classifications detailed below.

a Mapping by COBIT governance objectives

The COBIT framework defines five governance objectives (see Appendix A) to be applied in any IT organization and which are related to evaluation, direction, and monitoring. Their deployment implies ensuring that there is a governance structure capable of addressing the competencies related to IT governance that were mentioned above. These are the following ${ }^{20}$ :

- EDM01: Ensure Governance Framework Setting and Maintenance.

- EDM02: Ensure Benefits Delivery.

- EDM03: Ensure Risk Optimization.

- EDM04: Ensure Resource Optimization.

- EDM05: Ensure Stakeholder Engagement.

On this basis, each of the papers was assigned to one or more of the governance objectives (see Table 1) depending on its subject matter and the information offered, always with the consensus of the authors.

b Mapping by COBIT governance components

COBIT also defines a set of governance components (see Table 2) that must be ensured and managed by the organizational structures in order to establish, tailor, and sustain a governance system. Components are defined in COBIT as those factors that contribute to the proper development of IT governance activities. They can be of multiple types, acting individually or collectively, and may also be generic components or variants of the latter.

As in the previous case, these papers were read in full and assigned to one or more components, depending on their subject matter and the proposed solutions (see Table 3). These assignations were checked by all the authors, leading to the revision of dubious papers and to other changes these three individuals had agreed on.

Finally, primary papers within each objective were selected, offering the results that will be shown in the following sections.

TAB LE 1 Mapping by Control Objectives for Information and Related Technology (COBIT) objectives

\begin{tabular}{lcc} 
Objective & Reference & Number \\
EDM01 & $14,17,18,36-49$ & 17 \\
EDM02 & $36,40-42,45,48$ & 6 \\
EDM03 & $17,40,42$ & 3 \\
EDM04 & $36,39,42,43,45,48,50$ & 7 \\
EDM05 & $14,17,18,37-45,47-49,51$ & 16 \\
\hline
\end{tabular}

TA B LE 2 Control Objectives for Information and Related Technology (COBIT) governance components

\begin{tabular}{|ll}
\hline Component & Description \\
\hline Processes & A set of practices aimed at producing results that are useful to the organization's objectives. \\
\hline Organizational structures & Entities in charge of making key decisions in the organization. \\
\hline Principles, policies, and frameworks & Practices in terms of day-to-day management. \\
\hline Information & Information produced and used by the enterprise. \\
\hline Culture, ethics, and behavior & Sociocultural and ethical aspects that influence the interaction between individuals in the organization. \\
\hline People, skills, and competencies & Characteristics related to people and the interaction between them and their work. \\
\hline Services, infrastructure, and applications & Technology, applications, and any physical means that is part of the governance system. \\
\hline
\end{tabular}


TAB LE 3 Mapping by Control Objectives for Information and Related Technology (COBIT) components

\begin{tabular}{|ll|l}
\hline Component & Reference & Number \\
\hline Processes & $14,17,18,36-46,48,49,51$ \\
\hline Organizational structures & $14,17,36-49$ \\
\hline Principles, policies, and frameworks & 16 \\
\hline Information & $14,17,18,36-51$ \\
\hline Culture, ethics, and behavior & $14,17,18,36,38,39,43-45,47,48,50$ \\
\hline People, skills, and competencies & $17,36,39,45,48,49$ \\
\hline Services, infrastructure, and applications & $14,17,18,36,37,39,40,43-49$ \\
\hline
\end{tabular}

\section{6 | Results}

As may be observed in Figure 2, based on the results obtained, the highest concentrations of publications on GSD governance were in 2011, 2012, and 2015. It is worth noting the lack of publications on GSD governance after 2016. As regards the type of mapped publications, 10 journal papers (53\%) and nine conference papers (47\%) were mapped.

The overall results of the mapping are shown in Figure 3, which provides an overview of the frequency of publications by COBIT components and objectives, making it possible to differentiate between the main trends in GSD governance for the classified papers.

Detailed descriptions of the results obtained for each COBIT governance objective, along with those for the different components, will be provided in the following subsections. A detailed description of the results for both objectives and components can be found in Appendix C. These findings will serve to address the research questions, highlighting the most relevant publications and solutions dealt with in the literature studied.

\subsection{1 | COBIT governance objectives}

From the point of view of the COBIT objectives treated most widely in GSD governance (see Figure 4), the clearest trends in the literature were shown for EDM01 (89.47\%) and EDM05 (84.21\%). These objectives deal with the establishment of governance frameworks and collaboration between stakeholders, respectively. The category that is dealt with least in the mapped publications is that corresponding to risk assessment, EDM03 (15.79\%), whereas EDM02 (optimal value assurance on IT investments; 31.58\%) and EDM04 (resource assurance; 36.84\%) are balanced.

4

3

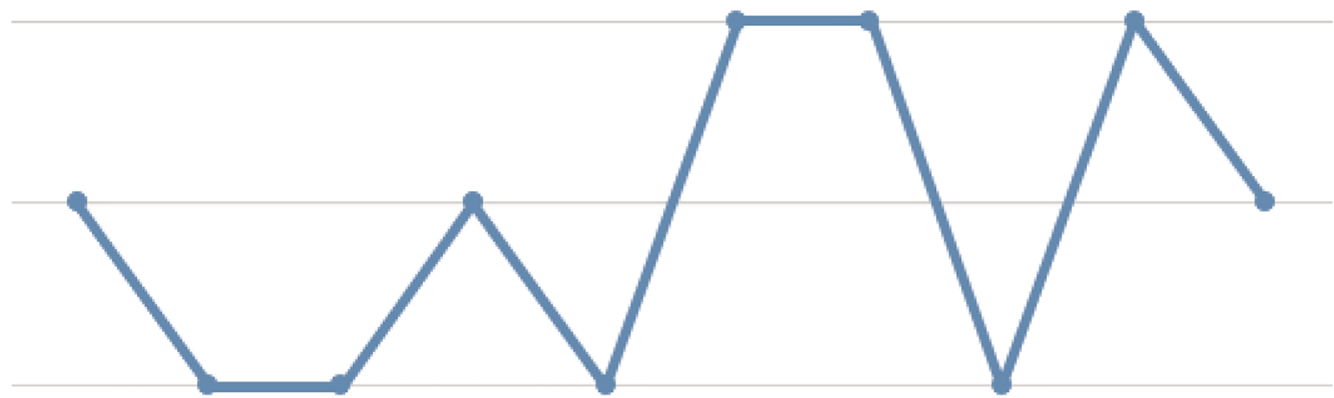

1

-

2

0

$\begin{array}{llllllllll}2006 & 2007 & 2008 & 2009 & 2010 & 2011 & 2012 & 2014 & 2015 & 2016\end{array}$

FIGURE 2 Trend in the number of publications per year 


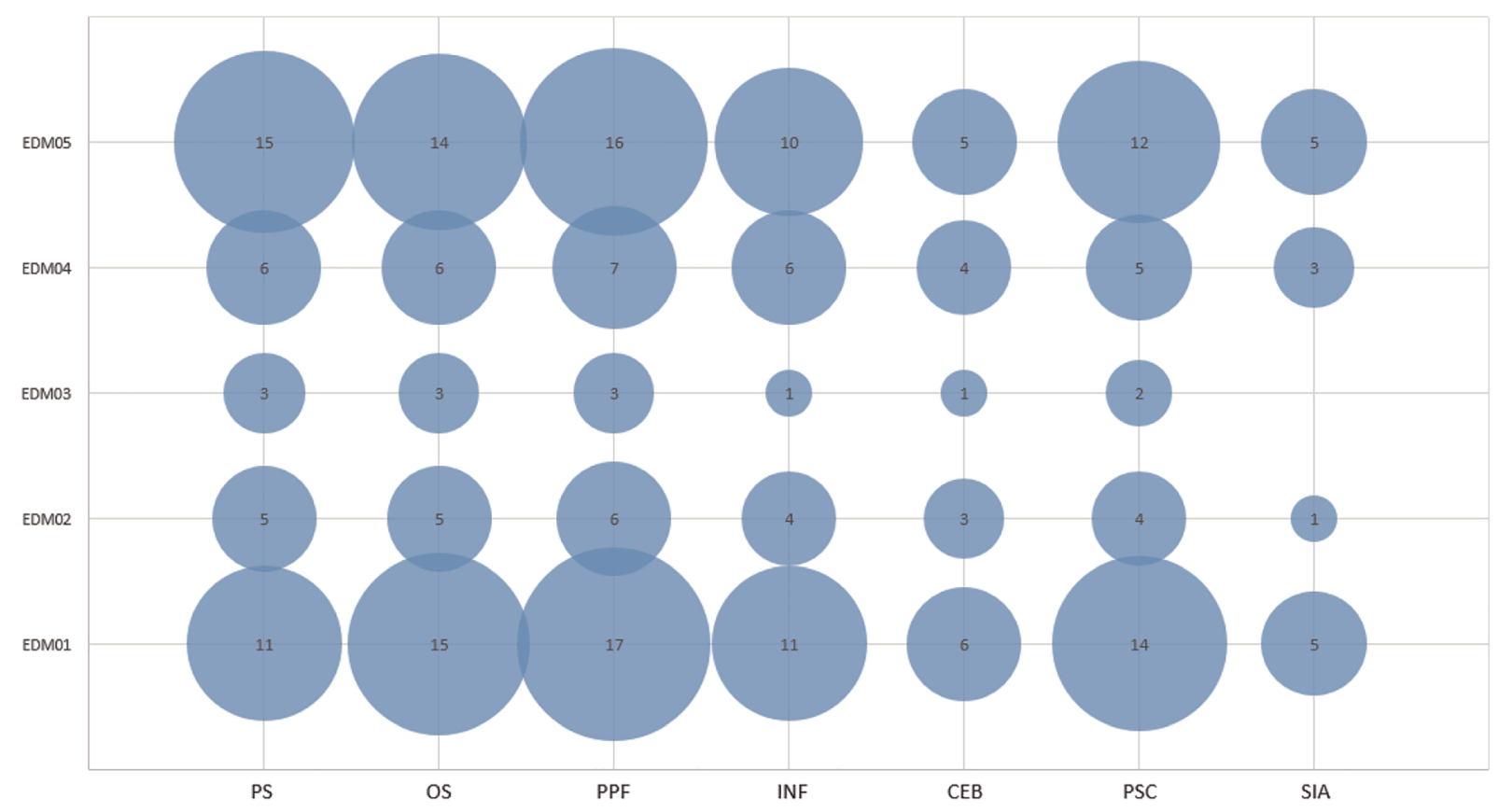

FIGURE 3 Control Objectives for Information and Related Technology (COBIT) objectives and components frequencies

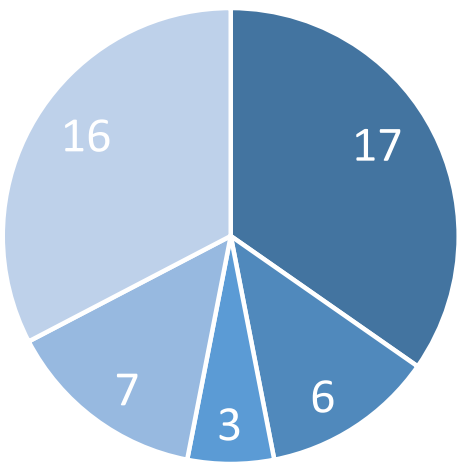

FIGURE 4 Frequency of each Control Objectives for Information and Related Technology (COBIT) objectives in the literature

- EDM01 EDM02 - EDM03 EDM04 EDM05

We will approach RQ1 by trying to study the state of the art for each of the COBIT objectives in greater detail. The main challenges and solutions will be addressed, highlighting the most relevant and recurrent issues treated in the literature.

a EDM01: Ensure Governance Framework Setting and Maintenance

The EDM01 objective is related to establishing and managing the governance structure of the IT organization, as well as to identifying strategies, responsibilities, missions, and business objectives. This was the objective addressed most in GSD governance literature, its being the one that defines the governance basis of an IT organization. A total of 17 papers (89.47\%) were mapped in this objective.

Regarding the literature related to this objective, Bavani ${ }^{40}$ presents different GSD governance patterns revealed through two case studies. Practices such as the definition of success parameters are emphasized, as are the periodical identification of both short- and long-term goals, and the awareness of common governance goals. As can be seen, not only the definition, but also the awareness of common objectives, is a responsibility usually attributed to the governance of an organization. This is even more relevant in distributed environments, if we consider how this lack of awareness is exacerbated by the distances that influence GSD.

On the other hand, Noll et al. ${ }^{17}$ propose a Capability Maturity Model Integration (CMMI)-style process area (the Global Teaming Model), which establishes objectives and practices to facilitate decision-making, stakeholder organization and communication, as well as measurement. The Global Teaming Model put forward can be seen as a governance model, as it presents governance activities that influence communication 
and knowledge management in distributed environments. At the same time, they also emphasize that GSD environments require explicit governance in order to avoid obstacles related to communication barriers.

Noordeloos et al. ${ }^{47}$ and Šablis and Šmite ${ }^{49}$ address the transition to agile development models in distributed environments, as well as the repercussions that governance has on the organization and collaboration between agile teams. In Šablis and Šmite, ${ }^{49}$ the influence that distance barriers have on the exchange of knowledge is shown. The correct organization of roles and responsibilities (a key factor supported by EDM01) is also highlighted by them. For their part, Noordeloos et al. ${ }^{47}$ show an organizational transition from Rational Unified Process (RUP) to Scrum, setting out the different changes necessary to ensure this process. Again, special relevance is attributed to business strategy definition, as well as to team structure and task allocation when dealing with this type of business transitions, which fall within governance responsibilities. In Vlietland and van Vliet, ${ }^{51}$ a conceptual governance framework for the management of Scrum teams is proposed, thus making significant progress in this field.

If we focus our attention on governance mechanisms, the work of Ali and Green ${ }^{38}$ identifies several key factors in IT governance, validated through empirical study: existence of ethics and compliance culture, corporate communication systems, greater involvement of senior managers, and use of a properly planned metrics system to measure performance and achievement of objectives. As explained, the use of well-known metrics facilitates monitoring and, therefore, aids the governance decision-making process. These metrics can have an adverse effect if they are not chosen judiciously; standard measures are thereby needed in order to optimize governance efforts. This is even more so in distributed environments, where physical, temporal, and cultural separation are common sources of risk that need to be measured.

Another work on governance mechanisms in GSD is Dubinsky et al. ${ }^{18}$ which also highlights the relevance of the aforementioned awareness about the goals and the organization. According to them, well-defined governance aligned with the business goals results in highly achieved goals. With respect to the use of tools that facilitate the task of governance, there is an evident lack of tools that automate the decision-making process and governance activities. We can therefore observe a possible research gap in this field if we look at the frequency of publications associated with the corresponding COBIT component.

Finally, Doležel and Buchalcevová, ${ }^{41}$ together with Ramasubbu and Balan, ${ }^{48}$ show progress from the practitioners' and researchers' points of view, respectively. Doležel and Buchalcevová ${ }^{41}$ presented a Test Governance Framework (TeGoF), where cultural factors were considered as a "potent and seminal factor" in the setting in which the framework was deployed. From the research point of view, Ramasubbu and Balan ${ }^{48}$ once again highlight the need for empirically proven dispersion metrics, as well as models for costs estimation, team organization, and definition of key processes.

The main issues addressed in this objective are summarized in Table 4, where those publications that address each of the issues are listed.

b EDM02: Ensure Benefits Delivery

EDM02 defines how to ensure profits by optimizing business value and by ensuring efficiency in the balance of costs and profits. With $31.58 \%$ of publications mapped in this objective, the main contributions in this field are highlighted as follows.

\section{TABLE 4 Main EDM01 issues}

\begin{tabular}{|c|c|}
\hline Issue & Related work \\
\hline Processes & 17,46 \\
\hline Decision-making & $17,18,36,43$ \\
\hline Stakeholders organization & 17 \\
\hline Communication and collaboration & $17,36,45,47$ \\
\hline Metrics & $17,36,46$ \\
\hline Business strategy & 43,45 \\
\hline Teams & $17,43,45-47,49$ \\
\hline Task allocation & 43,45 \\
\hline Knowledge management & $43,44,47$ \\
\hline Awareness & 18,38 \\
\hline Agile development & 45,47 \\
\hline Frameworks & 39,49 \\
\hline Ethics & 38 \\
\hline Culture & $17,36,39,45,48,49$ \\
\hline Tools & 18 \\
\hline Costs & 48 \\
\hline
\end{tabular}


In Ali and Green, ${ }^{38}$ the search for the most effective governance mechanisms is addressed not only in terms of performance but also of costs. Their work also highlights how an organization with ineffective governance is adversely affected by cost overruns that nullify its competitiveness.

From another perspective, in Noordeloos et al. ${ }^{47}$ the transition to agile methodologies is shown as a governance-level decision capable of reducing internal costs, thanks to improved communication between teams and increased flexibility in the way of working. Good communication therefore seems to be closely related to the reduction of costs in outsourcing, because solving communication problems increases performance and decreases internal costs, allowing organizations to "fully profit from the outsourcing benefits."

In Gewald and Helbig, ${ }^{42}$ the importance of relationship management as a key factor in governance models is highlighted by Gewald and Helbig, while, at the same time, four types of partnership relationships between IT service providers are shown to exist. These depend on whether the focus is on costs or competency. Thus, organizations that demand commodity IT services to automate rudimentary administrative processes look for the lowest possible cost; this relationship type is followed by that with providers (where a more expensive but higher quality IT investment is sought), then with partners (where the focus is not on the cost but on the business benefit derived from the IT investment), and finally, with advisors, where cost tracking is carried out, but as the least significant factor that is taken into account.

In a nutshell, and according to Johansson, ${ }^{43}$ cost control is an activity that must be carried out by IT governance. Those organizations that choose to decentralize their business require stronger governance structures that are able to offer this control in a tighter way. Although management should control costs at project level, governance should operate on an overview of these costs. Controlling the costs of the business operations carried out is therefore highly desirable in outsourced environments, where governance tasks tend to be more demanding.

In Table 5, a summary of the main issues addressed in this field is set out.

c EDM03: Ensure Risk Optimization

Let us now look at the most relevant publications for EDM03. This is the objective dealt with least, according to the mapping procedure, with only $15.79 \%$ of the total number of publications. The topics addressed here are those related to risk awareness, communication, identification, and management, ensuring that all these risks do not exceed the levels tolerated by the organization.

In Gewald and Helbig, ${ }^{42}$ they emphasize the idea that outsourcing entails risks, and they underline how organizational structures require effective actions to mitigate them. Although GSD governance must carry out a strategic management of the risks, the functional level will do it from a managerial perspective. As Gewald and Helbig point out, the interdependencies between tasks and their location in different distant sites imply a greater need for coordination, problem-solving, and mutual adjustment. This means that outsourcing generates benefits that can be limited, or strongly influenced, by the risks inherent to this business model.

It should be said that the work of Magnusson and Chou, ${ }^{44}$ defining as they do a compliance and risk framework for outsourced environments, deserves special mention. Based on the analysis method presented in Bass et al. ${ }^{52}$ and the set of risks defined by Ebert et al. ${ }^{53}$ for GSD projects (those related to efficiency, presence, talent and flexibility), they offer three types of perspectives on risk management: at the level of business, operational and project, trying to put forward a point of view closer to governance than that provided by previous pieces of work, which focused more on a management perspective. Finally, the framework described in this paper addresses two of the main challenges in outsourced GSD projects: on the one hand, the construction of an application system in a well-designated architecture and, on the other, the overcoming of sociocultural, temporal, and geographical barriers, together with the communication problems they entail.

Table 6 presents the main problems addressed in EDM03 and the publications related to them.

\section{d EDM04: Ensure Resource Optimization}

Ensuring the optimization of resources (people, processes, and technology), as well as guaranteeing their availability to support business objectives, is an essential aspect of IT governance; it is thus specified in the EDM04 objective, where 36.84\% of the publications were mapped.

The access to IT resources and the optimization in their use is a key factor in achieving competitive advantages. As Ali and Green ${ }^{38}$ explain, if available resources fall below expectations, outsourcing is a viable strategy for correcting such gaps in an enterprise. At the same time, they

\section{TABLE 5 Main EDM02 issues}

\section{Issue}

Competitiveness and competency

Cost reduction

Communication costs

Cost control

Outsourcing costs and benefits
Related work

36,40

36,45

47

43

38 
TABLE 6 Main EDM03 issues

Issue
Frameworks
Risk levels and architecture
Outsourcing distances
Communication and coordination
Risk management
Task allocation
Risk monitoring

highlight the responsibility of IT governance in the management of resources, and in their alignment with business objectives, identifying roles that will allow effective control over these.

In Srinivasan et al. ${ }^{50}$ relevant lessons learned from a relationship building workshop are presented, where overarching strategy, competence management, project planning, communication, and trust are shown as key factors in exploring relationships between distributed teams and in attempting to improve these. The management and optimization of human resources in distributed environments is faced with sociocultural, geographical, and temporal distances that make it difficult to build ties between work teams that operate in different countries and that need reliability if they are to cooperate more effectively. Making known who does what, as along with planning the hierarchy and formation of work teams properly, are tasks assigned to GSD governance. The influence of factors such as process awareness, trust, and cultural differences in relationships is also shown in Doležel and Buchalcevová. ${ }^{41}$

Sinha et al. ${ }^{54}$ highlight the need for more collaborative tools unified by a common framework. Such tools would provide more information on how resources are used and how they could be optimally assigned.

In summary form, Table 7 shows the most commonly treated topics in this field.

e EDM05: Ensure Stakeholder Engagement

The second objective as regards number of publications (84.21\%) is EDM05: ensuring participation and agreements with stakeholders. This objective details key aspects such as identification and engagement among key parties. In addition to that, communication and collaboration between well-defined stakeholders should be facilitated through appropriate governance efforts, especially in the context of GSD, where these factors may be hampered.

In Silveira and Prikladnicki, ${ }^{59}$ the definition of governance roles in communication between sites is addressed, as is the perception by their distributed companions of the availability of given individuals. Distances are shown as a barrier to knowledge exchange that affects the assigning of roles and responsibilities. It is also confirmed that distributed teams are not completely autonomous, because of knowledge dependencies.

According to Andriole, ${ }^{39}$ governance is evolving towards participatory models where both internal and external stakeholders influence strategic decisions. Collaboration between these stakeholders is now supported by acquisition, deployment, and technological support through cloud computing resources; at the same time, governance now focuses on productivity and partnership, as opposed to the old conception of it as a mechanism with a single function of standardization and control. As the Andriole points out, participatory governance recognizes the expansion in the number of stakeholders involved in governance, also acknowledging factors such as outsourcing or emerging technology.

The key factor of communication in distributed environments is a recurring theme among the different authors. Bannerman ${ }^{14}$ notes that governance plays a fundamental role in ensuring that relational mechanisms bring about proper collaboration and communication between dispersed stakeholders, also facilitating knowledge management. ${ }^{43,44}$ In Manteli et al. ${ }^{46}$ they reveal governance challenges related to business strategies,

\section{TABLE 7 Main EDM04 issues}

Issue

Alignment of resources with business objectives

Role identification

Policies and compliance

Metrics and monitoring

Human resources

Tools

\section{Related work}

38

38

38 
the composition and structuring of work teams and the localization of tasks. Some of the main problems identified in multisite software governance are information barriers, unbalanced team sizes, different hierarchical structures, roles between sites, or developmental reliance.

Governance frameworks are, therefore, required for the purpose of facilitating collaboration among stakeholders, identifying processes and offering recommendations in this domain. This was the work carried out by Noll et al. ${ }^{17}$ in their Global Teaming Model, described previously. As they mention, the opportunities to see how governance shapes the project are fewer owing to the lack of project encounters in distributed contexts. Individuals may also feel alienated from the projects in which they are involved. The objectives, practices, and subpractices offered by the proposed model seek to alleviate all these difficulties and enable cooperation between stakeholders.

According to Dubinsky et al. ${ }^{18}$ the problems associated with lack of awareness in GSD projects can be alleviated if team members meet with stakeholders regularly, sharing their vision and receiving feedback. Trying to hold more frequent meetings, or providing means of communication that facilitate collaboration between stakeholders, is part of GSD governance responsibilities. This idea was also presented in Bavani, ${ }^{40}$ a paper that recommends among its best practices that annual planning sessions between stakeholders should be encouraged.

In agile outsourcing research, Vlietland and van Vliet ${ }^{51}$ open the way for future work on the development of a governance framework to mitigate problems between distributed teams. A first step could be the search for improvements in communication, enabling reduction of the internal costs of the organization. ${ }^{47}$

When it comes to building and managing relationships, according to Gewald and Helbig, ${ }^{42}$ the desired levels of partnership between customers and IT service providers need to be defined. In this work, four levels of partnership are specified; their impact turns out to be of special significance in the model of governance to be adopted by the organization. On the other hand, in the work of Srinivasan et al. ${ }^{50}$ openness and trust are critical factors in the customer-supplier relationship. Stakeholder relationships must be characterized by timely and accurate communication and mutually beneficial relationships. The definition of collaborative processes between stakeholders, and the reduction of misunderstandings as regards requirements, are also significant recommendations to consider from a governance point of view.

Finally, Table 8 summarizes the main topics covered in this area.

\subsection{2 | COBIT governance components}

In this section, RQ2 will be addressed, discussing which particular governance components were the main solutions proposed for each COBIT objective in the mapped publications.

As shown in Figure 5, "principles, policies, and frameworks" (100\%) is the most common one, closely followed by "processes" (89.47\%) and "organizational structures" (84.21\%). Multiple practices and frameworks are proposed as solutions to typical problems in GSD governance; processes and governance structures that manage and execute them are also proposed. At the same time, "information" (63.18\%) and "people, skills, and competencies" (73.68\%) have close percentages located at a midpoint, whereas the components addressed least in the literature are "services, infrastructure, and applications" (31.58\%) along with "culture, ethics, and behavior" (31.58\%).

Figure 6, for its part, shows a detailed breakdown of the mapping by components, where we can observe the percentage of publications for each component and COBIT objective.

It should be noted, generally and transversally to the set of objectives, that all papers address the principles, policies, and frameworks component. This is because those papers that do not attempt to provide a framework at least seek to advise on best practices in governance issues. By contrast, services, infrastructure, and applications is the minority component in all the objectives, with the only exception being EDM03.

Let us therefore proceed to deal with the frequency of each of the components, objective by objective, discussing the most interesting aspects found after the mapping procedure.

\section{TABLE 8 Main EDM05 issues}

\begin{tabular}{|c|c|}
\hline Issue & Related work \\
\hline Collaboration and communication & $14,17,18,37,40,45,47-49$ \\
\hline Technological support and tools & 39 \\
\hline Involvement and partnership & 37,40 \\
\hline Knowledge management & 43,44 \\
\hline Teams & $17,18,38,43-45,47-49$ \\
\hline Roles & $43,44,47$ \\
\hline Frameworks & 17,49 \\
\hline Processes & $17,48,49$ \\
\hline Awareness & $17,18,48$ \\
\hline
\end{tabular}


FIGURE 5 Frequency of each Control Objectives for Information and Related Technology (COBIT) component in the literature

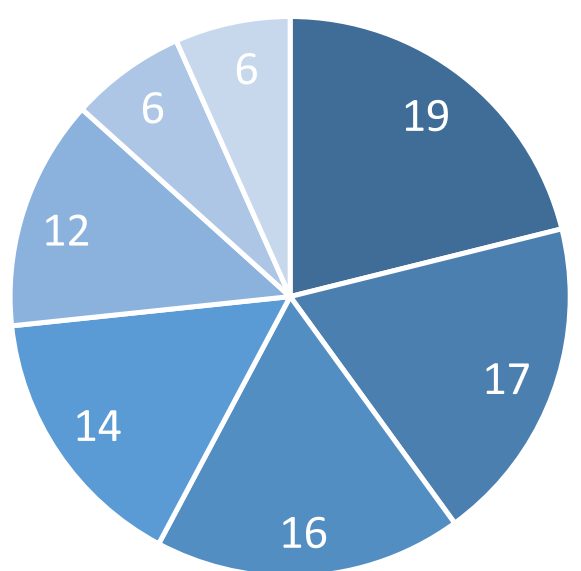

- Processes

- People, skills and competencies

n Culture, ethics and behavior

- Services, infrastructure and applications

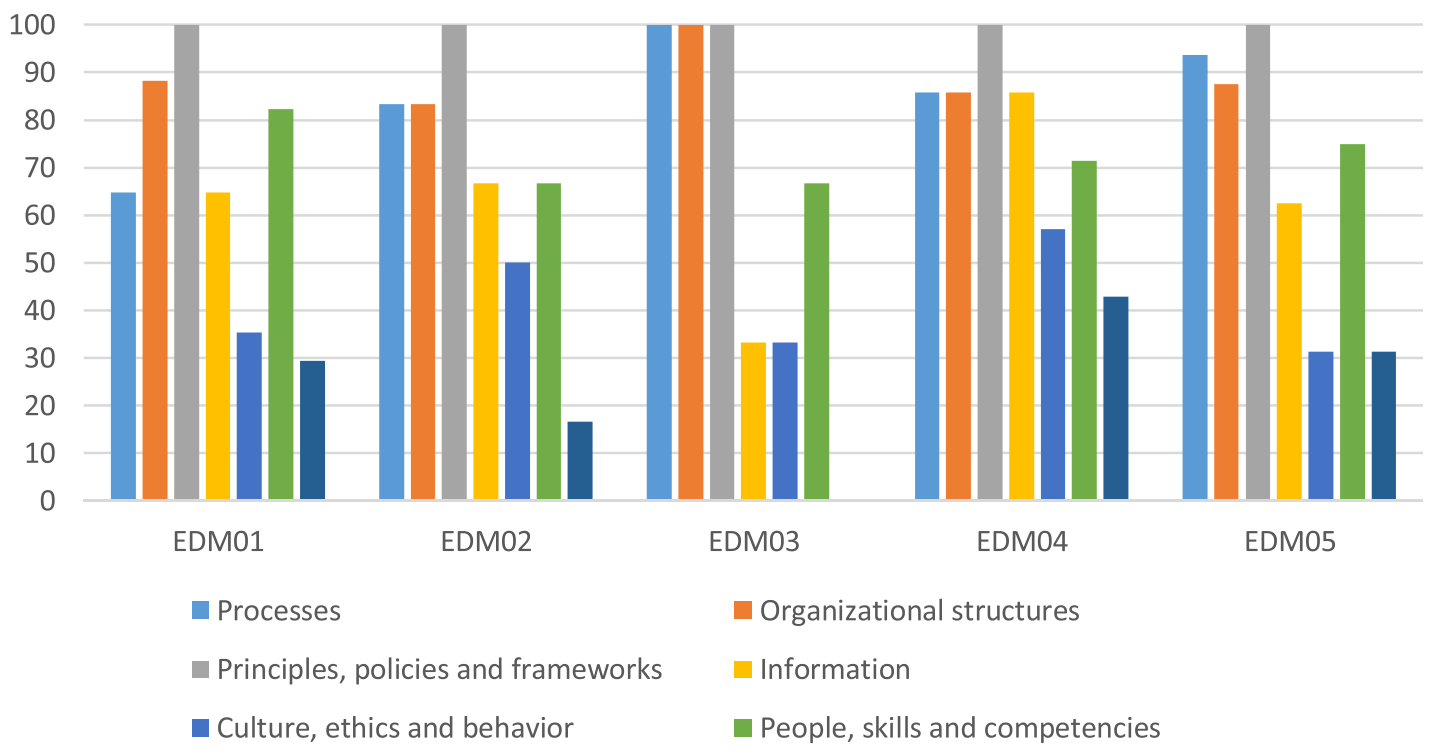

- Services, infrastructure and applications

FIGURE 6 Control Objectives for Information and Related Technology (COBIT) components frequency for each governance objective

a Processes

Regarding processes, the results show a greater amount of contributions in the literature for objectives EDM03 (100\%) and EDM05 (93.75\%). Most of the papers classified in EDM04 (85.71\%) and EDM02 (83.33\%) also address this component, with EDM01 (64.71\%) being the objective with the least emphasis on process-related solutions.

In terms of research gaps, and on the basis of these results, it is possible to go into greater depth regarding aspects related to the establishment of processes for the definition, management, and maintenance of governance frameworks. Processes related to the analysis and establishment of governance requirements, or to the specification of authorities and responsibilities to achieve organizational goals and objectives, are well suited to this field.

From the point of view of GSD governance, it would be important for these processes to address the perspectives of outsourcing, either by defining new types of processes related to exclusive GSD activities or by shifting existing frameworks towards a new, broader perspective. 
b Organizational structures

Organizational structures have great significance in all the objectives: EDM03 (100\%), EDM01 (88.23\%), EDM05 (87.5\%), EDM04 (85.71\%), and EDM02 (83.33\%). The role they play in governance efforts is essential, as is noted in the literature.

Although this component is addressed in broad terms, we believe that the emphasis on outsourced environments remains limited. Criteria associated with decision-making or means to simplify this process in distributed settings are good candidates for future research. In addition, we believe that studying the variation between in-house and outsourced organizational business structures, as well as the transition from one to the other, could be of great interest to practitioners.

c Principles, policies, and frameworks

The results relating to this component are the most significant, with $100 \%$ of publications mapped to all the objectives. To understand this, it is necessary to consider the state of the art and what solutions have been proposed in each of the previously mentioned pieces of work. As can be seen, GSD governance is still a very recent field, where some frameworks have been proposed in an effort to normalize this area. On the other hand, some publications do not propose frameworks; instead, they offer practices or policies to be adopted in the attempt to solve problems related to this domain.

The fact that this component has been addressed in each and every publication therefore also reveals the relevance of this research area. At the same time, we believe that the standardization of GSD governance should be done progressively, through new frameworks and standards that take on this task, so future work on this issue is still needed in the endeavor to increase the maturity of GSD governance.

d Information

The results obtained for this component highlight a greater emphasis on EDM04 (85.71\%), followed by EDM02 (66.67\%), EDM01 (64.71\%), and EDMO5 (62.5\%). In this case, EDM03 (33.33\%) is the objective that addresses the information least.

Information is a transversal component to all the objectives, and it must flow from the operational to the strategic levels, thus facilitating decision-making processes and providing data on the current status of the different sites of the organization, if we focus on GSD.

How information should be used to establish and maintain governance components (EDM01), ensure benefits (EDM02), or manage stakeholder relationships (EDM05) is a gap that could provide many opportunities for future research. Focusing on GSD, how information is distributed between sites, and how it can be leveraged to improve outsourced business processes, is also a necessary gap to be addressed.

Finally, if we look at risks (EDM03), where there seems to be a greater lack of solutions related to information, it is useful to know what risks are inherent to information management, as well as how information can be used to reduce risks. It would be interesting to know if there are any risks associated with the treatment and dissemination of information that are accentuated due to the influences exerted by distances between sites.

e Culture, ethics, and behavior

This is one of the least discussed components in the literature, and it is here that we have observed a large research gap that needs to be addressed. Only in EDMO4 (57.14\%) and EDMO2 (50\%) are there at least 50\% of the publications that deal with this topic. However, in EDM01 (35.29\%), EDM03 (33.33\%), and EDM05 (31.25\%), there is a great lack of publications that relate governance to culture, ethics, and behavior.

The results for this component are worth paying attention to, given the importance of cultural aspects in stakeholder communication, participation, and agreements. It would also be interesting to study how governance structures should adapt to the inherent multiculturalism of GSD environments when making decisions or establishing governance schemes.

Understanding what risks are inherent to cultural differences is a gap that would also be interesting to tackle, as would knowing how these risks can be reduced. Given the importance and influence of sociocultural distances in GSD environments, future research on this topic will be needed.

f People, skills, and competencies

Governance competencies relating to people, skills, and competencies have been addressed to a great extent in all objectives: EDM01 (82.35\%), EDM05 (75\%), EDM04 (71.43\%), EDM02 (66.67\%), and EDM03 (66.67\%).

The greatest deficiencies are found in the objectives referring to insurance of benefits and treatment of risk. Opportunities therefore arise to delve more deeply into how the competences of individuals have an impact or can be exploited when it comes to optimizing business value or 
reducing risks. The study of the relationship between skills and cultures as regards governance decision-making in GSD settings would be equally interesting.

g Services, infrastructure, and applications

Last of all, we come to the objective related to the support of services, infrastructure, and applications. This is the component of all objectives that is dealt with least of all, a fact that highlights and proves the need for further research on this topic.

The field that alludes most frequently to this component is EDM04 (42.86\%), followed by EDM05 (31.25\%), EDM01 (29.41\%), and EDM02 (16.67\%). No publication referring to EDM03 (0\%) proposes solutions related to this component, so future proposals are required.

Instead of proposing solutions for each of the COBIT objectives, we believe that the solutions related to this component should be transversal for each of the objectives. Tools and technological support are needed to allow the monitoring and management of benefits (EDM02), risks (EDMO3), and resources (EDM04), offering an overview of the organization and thus facilitating decision-making and governance efforts. This component also plays a key role in relationships between stakeholders (EDM05), where geographical distances force communication through tools that should ease remote cooperation and collaboration.

It is thus clear that tools, applications, or services are necessary for managing and monitoring any type of information, metric, process, or resource inherently related to the IT business; they optimize decision-making and operations in outsourcing settings where this tracking gets more complex.

\section{4 | SOLUTIONS AND CHALLENGES}

In the following subsections, we will summarize the main solutions proposed in the literature, also pointing out those challenges that still remain as research gaps to be addressed in future research. Both solutions and challenges will be presented in terms of COBIT components, in an effort to provide an organized perspective on GSD governance research.

\subsection{Solutions}

- Processes

In this domain, we highlight the solution provided by Noll et al. ${ }^{17}$ with their Global Teaming Model, in which a formal set of processes for GSD governance is set out in detail. In this model, the highest level governance objectives are based on the management of global projects and locations. Specific practices and subpractices, along with recommendations, are defined to achieve them. The distinction among the practices "global task management," "knowledge and skills," "global project management," "project management," "operational procedures," and "collaboration between locations" are perfectly mappable onto COBIT objectives and components, which makes this model a good example to follow when evaluating the fulfillment of governance tasks in real environments.

Also worth highlighting is the explicit definition offered by Bannerman ${ }^{14}$ of the competences related to governance processes; they include accountability, decision-making, monitoring or planning, and among others.

The relationship management between customer and service provider through joint processes and their role in the organization is highlighted in Gewald and Helbig, ${ }^{42}$ where processes related to strategy, strategic risk management, and annual planning are attributed to governance.

\section{- Organizational structures}

Regarding this component, Andriole ${ }^{39}$ highlights the current trend towards participatory governance structures, as well as the need to leverage cloud resources to support communication and collaboration. Bavani, ${ }^{40}$ for his part, differentiates between three governance patterns: delegate, collaborate and multipartner, which define how governance structures operate in distributed settings. As this work and others such as Johansson ${ }^{43}$ indicates, a key to success in GSD governance comes from establishing strong local governance. Specialized governance members can, at the same time, help to solve technical governance problems that could have a negative impact on global teams. ${ }^{40}$

- Principles, policies, and frameworks

Although many good practices related to GSD governance have been proposed in literature, we will emphasize those that have been put forward as frameworks or conceptual models. The main solutions proposed in this domain are frameworks related to risks and compliance, ${ }^{44}$ coordination of agile teams, ${ }^{51}$ test governance, ${ }^{41}$ outsourced partnership, ${ }^{42}$ global teaming processes,,${ }^{17}$ and tool integration. ${ }^{54}$ 
- Information

Focusing on information, in Manteli et al. ${ }^{45}$ an analytical method is proposed for the analysis of the effect of governance decisions on transactional memory systems. It also provides useful information on the role of boundary spanners or expertise location. Manteli et al. ${ }^{46}$ for their part, offer a governance structure for distributed environments where processes to carry out this work and which are focused on knowledge management are also described.

Another noteworthy contribution is the analysis of knowledge networks carried out in Silveira and Prikladnicki ${ }^{59}$; they identify governance roles, as well as issues such as the frequency of knowledge exchange between them. They state that the independence of distributed sites is limited by knowledge dependencies.

- Culture, ethics, and behavior

Regarding culture in GSD governance, we will highlight some of the governance processes and subprocesses described in Noll et al. ${ }^{17}$ such as creation of specific roles, relationships, and rules to reduce cultural distance; identification of cultural requirements for each team; training in the cultures of virtual teams; and coverage of knowledge transmission needs, considering cultural or linguistic problems that may arise or ensuring awareness of cultural profiles.

In similar vein, in the work of Noordeloos et al. ${ }^{47}$ daily communication is highlighted as a means to increase trust between distributed teams. Other benefits associated with this communication routine are the reduction of misunderstandings, work synchronization, task tracking, and awareness. They relate these communication practices directly to the governance competencies in charge of task allocation and team composition.

- People, skills, and competencies

According to Srinivasan et al. ${ }^{50}$ relationships between people in outsourced environments are related to key factors such as overarching strategy, communication, competence management, project planning, and trust. Some of the main opportunities for GSD governance improvement lie in the development of leadership, strategic cocreation, and the establishment of competitive engines between sites.

In Ali and Green, ${ }^{38}$ they find a positive correlation between effective IT governance and mechanisms such as the existence of a compliance culture, corporate communication systems, suitable performance measurement, and greater involvement of senior management. The importance of specific governance roles is also highlighted, as in Silveira and Prikladnicki, ${ }^{59}$ where the relevance of roles that are in charge of maximizing communication between distributed teams is presented as a key factor in outsourcing settings.

- Services, infrastructures, and applications

Solutions found for this component are few and far between. The conceptual architecture proposed by Sinha et al. ${ }^{54}$ for establishing a framework related to tool integration and collaboration enabling is a contribution that is worthy of mention.

\subsection{Challenges and open issues}

Now that the main solutions proposed in GSD governance have been analyzed, we need to consider in what fields there are still research gaps that need to be addressed and give thought to how the search for new solutions could be approached. To reveal these gaps, we took as our basis both the results of our mapping and what has been stated in the literature, identifying those objectives and components that had been dealt with least and that therefore offer opportunities for further, more in-depth research in future work.

The following points, then, outline some still-open issues to be considered in future work related to GSD governance.

\section{- Processes}

Although many processes and practices have been recommended in the quest to facilitate GSD governance efforts, the definition of a common standard process model that could be integrated into other known ones is particularly important if GSD governance practices are to be standardized. As explained in Ramasubbu and Balan, ${ }^{48}$ it must be considered that there are many processes that do not arise in colocated environments but which are crucial for GSD teams.

At the same time, a complete transition and redefinition of conventional processes towards outsourced settings is also needed in future research. Sriram and Mathew ${ }^{55}$ indicate the need to study the particular problems associated with governance and related processes that 
undermine the performance of global projects. A reference example when addressing this problem is the Global Teaming Model defined in Noll et al. ${ }^{17}$

Thus, the main challenges concerning GSD governance processes are, therefore, their unification and standardization and the adaptation of existing local processes to those of GSD governance, along with the problems resulting from their inadequate management, which could have even worse consequences than in allocated development in terms of costs and effort.

- Principles, policies, and frameworks

Despite this being the component dealt with most in the literature, we reiterate the need for frameworks that unify the existing knowledge in GSD governance, considering all the dimensions (strategies, benefits, risks, resources, and stakeholders) involved in this labor. The importance of these being tested by practitioners should not be forgotten: frameworks and models tested in real settings that address all the objectives related to GSD governance will increase the sturdiness of this domain.

If we explore the multicultural nature of GSD, the establishment of policies and working principles among teams comprising multiple nationalities is equally important. In fact, governance frameworks that reflect optimal means of communication and collaboration depending on the cultures involved in projects could be developed using these principles as a basis.

Not only the development but also the implementation of frameworks common to different sites is, therefore, a challenge to be addressed from the perspective of GSD governance, given the variety of working methods and business situations.

- Information

Information about the organization's internal processes is essential, as is defining and approving objectives, roles, and frameworks for proper knowledge management. Knowledge sharing is closely linked to communication and awareness, because it must be transmitted and assimilated.

From the point of view of knowledge, governance must take charge of the policies adopted in order to manage this information. The adoption of governance models must be in line with how knowledge is wished to be distributed. In turn, roles exclusively dedicated to managing knowledge and mediating between stakeholders (a similar approach to the boundary spanners described in Manteli et al. ${ }^{45}$ ) could be useful in guaranteeing a proper knowledge flow from operational levels to decision-making structures.

Likewise, the acquisition and support of high-level knowledge used for strategic decision-making could be favored by employing extensive data repositories, as well as by standardized and properly defined Big Data solutions.

It should also be said that the usefulness of metrics and the need for these is a recurring theme in literature ${ }^{17,36,46}$; after all, they offer a snapshot of the current state of the organization from multiple perspectives and dimensions. From a governance point of view, metrics must be established by governance structures, which also need to define how the metrics are applied in the task of gathering information and of improving the overall business performance.

How resources are harnessed, the internal and external costs, the quality of communication and cooperation between users, or the impact of sociocultural distances are some of the many factors that can be considered for measurement and that fall within GSD governance duties. The use of well-known metrics eases the governance decision-making process and assists in the monitoring of the objectives to achieve, although it should be noted that if metrics are poorly designed or incorrectly used, this may have a negative impact on the organization.

On this basis, some of the inherent difficulties as regards how information is managed and, principally, governed in distributed environments are the equal distribution of information among sites, the formation of specific roles for this work, and an agreement as to common metrics with which to assess the status of organizations, projects, teams, or sites.

- Culture, ethics, and behavior

Culture and trust have a great influence on organizational decisions, as well as on the relationship building between stakeholders. These problems increase with business distribution, reducing trust and widening sociocultural differences. There is therefore a need to know how cultural differences or lack of trust really influence GSD governance efforts, and thought should be given as to how these problems can be overcome.

It would also be interesting to apply knowledge regarding the features of the different cultures to the construction of virtual teams or to consider these features in an effort to strengthen the creation of links between stakeholders, enhancing mutual trust. A starting point could be work such as that done by Hofstede, ${ }^{56}$ which endeavors to quantify cultural differences, trying to apply technology that can take advantage of these kinds of social studies in governance decisions that are related to cooperation between stakeholders.

Closely related to culture, awareness plays an essential role in GSD governance duties. ${ }^{17,18,38,48}$ The decentralization of software development has meant inherent changes in business hierarchies and in role definition. Just as mediators are required between distributed work teams, specific information sources or roles are also required to raise awareness about who does what or to identify what the common objectives are and what their current status in the project development is. 
Decentralization reduces awareness of common goals and hinders development if it is not known who to turn to in response to particular situations or issues. Hence, the importance, whether on the part of certain people or sources of information, of raising awareness of business processes or of the state of common goals in the organization. Establishing the necessary means of developing awareness is a task that needs to be addressed by the governance structure.

We can conclude that the main handicaps that this COBIT component implies are the strengthening of awareness regarding the state of the organization and the projects between sites, along with the need for guides that, from the standpoint of governance, would us to discover which cultures together provide better outcomes. Assessing the competencies to be covered by specific roles associated with these problems could, therefore, be a complex endeavor.

- People, skills, and competencies

Redefining current roles in organizations and defining new ones can be a key factor in GSD governance. In fact, from a managerial point of view, this is a research field that has evolved considerably in recent years, with many publications addressing the creation of specific roles for decentralized enterprises, also studying their effectivity. ${ }^{36,47}$ However, in terms of governance, there is a lack of a clear definition of roles and their functions in relation to the administration of outsourced processes or resources.

Governance roles are needed. Organizational structures are in charge of approving roles responsible for raising awareness about the state of projects; individuals in charge of managing specific risks in outsourced environments, such as communication and collaboration between teams or roles aimed at using specific metrics to evaluate trends and act accordingly, are some of the ideas that could be further discussed in the near future. The work of Ali and Green ${ }^{38}$ could be taken as a reference when addressing this topic.

One of the main objectives in governance tasks is to determine and approve roles, responsibilities, and authorities for decision-making. From a communication point of view, approved governance roles are needed if there is to be better communication between stakeholders, distributed work teams, and governance structures. These roles take on great importance in the context of GSD; this is even more so in agile environments ${ }^{49}$ where continuous communication is a key factor in ensuring the success of the projects.

The geographical and time separation between stakeholders, in combination with the trend towards participatory governance models, ${ }^{39}$ are key factors that increase the importance of communication and the means available for decision-making. Relational mechanisms, for their part, are elements that take on the task of facilitating interaction and communication between stakeholders and that are intimately linked to the governance structure. ${ }^{14}$

If we, therefore, focus on challenges related to this component, in addition to normalized methods for both the definition of communication policies and the communication and assurance of business strategies, there are notable gaps in the context of GSD, in which an awareness of policies and strategies may be scattered and diffuse owing to the phenomenon of remoteness.

- Services, infrastructures, and applications

Although there are many tools proposed for management purposes, there is a lack of technological resources that would facilitate strategic decision-making ${ }^{18}$ and governance duties, in general. Tools designed to ease governance, providing high-level information, are necessary in the governance domain. In terms of distributed enterprises, standard integration methods between tools will need to be established, as will the correct use of cloud resources, whose importance is highlighted in Andriole. ${ }^{39}$

With regard to the inherent difficulties linked to this component, centralization is a complex task between sites that have different technologies and infrastructures, in addition to training in the technologies adopted.

\section{5 | VALIDITY THREATS}

In this section, we will detail the threats to validity that may have influenced our study, based on those set out in Wohlin et al. ${ }^{57}$; we will also outline how we have tried to minimize their effect.

\section{- Construct validity}

The choice of a suitable search string that allows a compilation of as many of the most representative primary studies as possible is a threat to construct validity that should be considered. In an attempt to avoid the risk of overlooking relevant publications owing to the particular search terms chosen, several tests were conducted with different variations of search strings until the most representative and least noisy string was found. To ensure that it worked properly, we took several relevant publications in the domain as a reference and ensured that they appeared in the search results, noting also that publications outside the domain of our discipline did not appear among the publications found. 
Regarding the chosen sources, we used Scopus, IEEE Digital Library, and ACM Digital Library as the primary search sources, as they are the largest and most extensive research databases in our field. In addition, COBIT 2019 was chosen as the classification framework, due to its relevance in the field, its being an updated framework, and its extensive use in the professional field. Besides these advantages, the way in which its governance objectives are framed was a prominent factor in facilitating the classification procedure.

Finally, one of the most common problems we faced in this study was the correct differentiation between governance and management competencies in papers where this distinction was not completely clear. This confusion also came about in publications with themes that were transversal both to governance and to management domains. In our case, COBIT served as a means of ensuring that there were no misunderstandings in this differentiation. As it explains, although governance objectives correspond to the EDM domain (evaluate, direct, and monitor), management objectives are grouped in APO (align, plan, and organize), BAI (build, acquire, and implement), DSS (deliver, service, and support), and MEA (monitor, evaluate and assess). ${ }^{20}$ Thus, following these guidelines and based on the definitions of the objectives and their competencies, this differentiation was achieved properly.

- Internal validity

Internal validity is affected by the process of extraction, analysis, and classification of publications.

With respect to the process carried out, first of all, a person was in charge of the extraction, analysis, and mapping of the publications, work that would later be reviewed by two other people who were responsible for evaluating this process, as well as for carrying out the mapping of any publications which in the first instance had been uncertain. This evaluation was undertaken individually by each of the subjects, ensuring impartiality and individuality in the decisions. Finally, the three people involved would inform each other on the decisions they had taken, so as to ensure that a definitive impartial classification was carried out. In decisions where there were discrepancies, a majority of at least more than half of the authors was needed in order for a final assignation to be made. For more information on the outcome of these assignations, see Appendix D.

In addition, the selection of inclusion, exclusion, and quality assessments was individually drafted and then pooled among the authors to ensure that the publications extracted were of real relevance in the field.

Although it was initially planned to consider only JCR publications, or publications belonging to international congresses, this idea was discarded, in the endeavor to compile as many papers as possible.

- Conclusion validity

To ensure the conclusion validity, and therefore the reproduction of a similar mapping with the same results, each of the steps carried out in the mapping process was expressly described. In addition to that, the mapping of the publications was carried out with the consensual decisions of three people, thereby attempting to reduce as much as possible the subjectivity in the assignation of papers to objectives or categories.

- External validity

With regard the generalization of the results obtained, it is not possible to make a complete extrapolation of them, both because of the possible existence of works that deal with GSD governance in an indirect way and that were not found with the search terms used and because of the possible loss of some publications present in the gray literature, which has not been contemplated. Despite this, we believe that the study provides an adequate overview of the state of the art of GSD governance, showing that there is a significant lack of publications in this area in major databases such as ACM, Scopus, and IEEE.

\section{6 | CONCLUSIONS}

The results of this systematic mapping of literature have allowed us to discover the state of the art in GSD governance, describing the main solutions and objectives pursued in this domain. The use of COBIT 2019 as a reference framework with which to classify publications on GSD governance has allowed us to identify multiple research gaps in this area that may be addressed in future research. Specifically, aspects related to metrics, tools, processes, awareness, knowledge, culture, trust, and roles still need to be dealt with in greater depth. The importance of governance in distributed development has been stressed, given the organizational, management, and governance challenges that GSD currently poses. Addressing these challenges could, therefore, be considered a goal to be achieved by the GSD community in future research.

We believe that the results obtained from this study may have important repercussions for both researchers and practitioners. From a research point of view, the state of the art of GSD governance has been studied, showing research gaps related to COBIT objectives and components and flagging up issues that are still open and that should be addressed in future work. One opportunity that the results obtained provide is the possibility of attempting to discover whether this is the case of the less frequently researched governance objectives and components 
because they really do not require more in-depth research or whether this absence of research is owing to a lack of awareness of the GSD governance state of the art.

It should also be acknowledged that the impact that the least addressed COBIT objectives have on industry will need to be validated. From the practitioners' point of view, they will be able to contrast the results with the objectives applied in their organizations, thus attempting to detect opportunities for improvement in this field by adopting best practices that are already present in the literature. Moreover, some of the challenges to which the results obtained in this study may lead are a discovery of the cause and relevance of the most common COBIT governance components and objectives in organizations, in addition to an attempt to discover the advantages that those less frequently addressed would have for practitioners.

We believe that knowing the current state of art in GSD governance will provide a promising and challenging future in this recent and scarcely treated domain, giving rise to new research opportunities and solutions.

\section{ACKNOWLEDGEMENTS}

This work has been funded by the G3SOFT project (Consejería de Educación, Cultura y Deportes de la Junta de Comunidades de Castilla La Mancha, y Fondo Europeo de Desarrollo Regional FEDER, SBPLY/17/180501/000150) and BIZDEVOPS-GLOBAL project (Ministerio de Ciencia, Innovación y Universidades, y Fondo Europeo de Desarrollo Regional FEDER, RTI2018-098309-B-C31).

\section{ORCID}

Antonio Manjavacas (D) https://orcid.org/0000-0001-7334-1074

Aurora Vizcaíno (D) https://orcid.org/0000-0002-2072-5581

\section{REFERENCES}

1. Ebert C. Global Software and IT: A Guide to Distributed Development, Projects, and Outsourcing. John Wiley \& Sons; 2011.

2. Damian D, Moitra D. Guest editors' introduction: global software development: how far have we come? IEEE Softw. 2006;23(5):17-19. https://doi. org/10.1109/MS.2006.126

3. Ågerfalk PJ, Fitzgerald B, Holmström Olsson H, Conchúir EÓ. Benefits of global software development: the known and unknown. In: Making Globally Distributed Software Development a Success Story; 2008:1-9.

4. Conchúir EÓ, Ågerfalk P, Olsson H, Fitzgerald B. Global software development: where are the benefits? Commun ACM. 2009;52:127-131, https://doi. org/10.1145/1536616.1536648

5. Herbsleb J, Mockus A, Finholt TA, Grinter RE. 'An empirical study of global software development: distance and speed', presented at the Proceedings - International Conference on Software Engineering, 2001, pp. 81-90. https://doi.org/10.1109/ICSE.2001.919083.

6. Olsson H, Conchúir EÓ, Ågerfalk P, Fitzgerald B. 'Global software development challenges: a case study on temporal, geographical and socio-cultural distance', presented at the Proceedings - 2006 IEEE International Conference on Global Software Engineering, ICGSE 2006, 2006, pp. 3-11. https:// doi.org/10.1109/ICGSE.2006.261210.

7. Jolak R, Wortmann A, Chaudron M, Rumpe B. Does distance still matter? Revisiting collaborative distributed software design. IEEE Softw. Nov. 35(6): 40-47. https://doi.org/10.1109/MS.2018.290100920

8. Ulhaq S, Raza M, Zia A, Khan MNA. Issues in global software development: a critical review. JSEA. 2011;4:590-595.

9. Noll J, Beecham S, Richardson I. Global software development and collaboration: barriers and solutions. ACM Inroads. 2011;1(3):66-78. https://doi. org/10.1145/1835428.1835445

10. Alzoubi YI, Gill A. Agile global software development communication challenges: a systematic review. In: PACIS; 2014.

11. Dumitriu F, Oprea D, Mesnita G. 'Issues and strategy for agile global software development adoption', Proceedings of the World Multiconference on Applied Economics, Business and Development, 2011:37-42

12. Vallon R, da Silva Estácio BJ, Prikladnicki R, Grechenig T. Systematic literature review on agile practices in global software development. Inf Softw Technol. 2018;96:161-180. https://doi.org/10.1016/j.infsof.2017.12.004

13. Ebert C, Kuhrmann M, Prikladnicki R. 'Global software engineering: evolution and trends', in 2016 IEEE 11th International Conference on Global Software Engineering (ICGSE), 2016, pp. 144-153. https://doi.org/10.1109/ICGSE.2016.19.

14. Bannerman PL. 'Software development governance: a meta-management perspective', in 2009 ICSE Workshop on Software Development Governance, 2009, pp. 3-8. https://doi.org/10.1109/SDG.2009.5071329.

15. Chulani S, Williams C, Yaeli A. 'Software development governance and its concerns', in Proceedings of the 1st International Workshop on Software Development Governance, New York, NY, USA, 2008, pp. 3-6. https://doi.org/10.1145/1370720.1370723.

16. Ericsson M. 'The governance landscape: steering and measuring development organizations to align with business strategy', 2007.

17. Noll J, Beecham S, Richardson I, Canna CN. 'A global teaming model for global software development governance: a case study', presented at the Proceedings - 11th IEEE International Conference on Global Software Engineering. ICGSE. 2016;2016:179-188. https://doi.org/10.1109/ICGSE.2016.33

18. Dubinsky Y, Ravid S, Rafaeli A, Bar-Nahor R, 'Governance mechanisms in global development environments', in 2011 IEEE Sixth International Conference on Global Software Engineering, 2011, pp. 6-14. https://doi.org/10.1109/ICGSE.2011.28.

19. ISACA, COBIT 2019 framework. Introduction and Methodology. ISACA, 2018.

20. ISACA, COBIT 2019 framework. Governance and Management Objectives. ISACA, 2018.

21. Ahmed Z, Mansor Z, Ahmad K. An analysis of knowledge management challenges in agile global software development. J Telecom Electron Comput Eng. 2017;9(3-4 Special Issue):63-66.

22. Anwar R, Rehman M, Wang KS, Hashmani MA. Systematic literature review of knowledge sharing barriers and facilitators in global software development organizations using concept maps. IEEE Acc. 2019;7:24231-24247. https://doi.org/10.1109/ACCESS.2019.2895690 
23. Zahedi M, Shahin M, Ali Babar M. A systematic review of knowledge sharing challenges and practices in global software development. Int J Inf Manag. 2016;36(6, Part A):995-1019. https://doi.org/10.1016/j.ijinfomgt.2016.06.007

24. Rashid N, Khan SU. Agile practices for global software development vendors in the development of green and sustainable software. J Softw Evol Process. 2018;30(10). https://doi.org/10.1002/smr.1964

25. Akbar MA, Nasrullah MS, Ahmad J, Maqbool A, Abbas K. 'Investigation of project administration related challenging factors of requirements change management in global software development: a systematic literature review', presented at the 2018 International Conference on Computing, Electronic and Electrical Engineering, ICE Cube 2018, 2019. https://doi.org/10.1109/ICECUBE.2018.8610966.

26. Anwer S, Wen L, Wang Z. 'A systematic approach for identifying requirement change management challenges: preliminary results', 2019, pp. 230-235. https://doi.org/10.1145/3319008.3319031.

27. Nicolas J, Carrillo De Gea JM, Nicolas B, Fernandez-Aleman JL, Toval A. On the risks and safeguards for requirements engineering in global software development: systematic literature review and quantitative assessment. IEEE Acc. 2018;6:59628-59656. https://doi.org/10.1109/ACCESS.2018. 2874096

28. Akbar MA, Sang J, Khan AA, Shafiq M. Towards the guidelines for requirements change management in global software development: client-vendor perspective. IEEE Acc. 2019;7:76985-77007. https://doi.org/10.1109/ACCESS.2019.2918552

29. Azeem Akbar M, Sang J, Khan AA, et al. Success factors influencing requirements change management process in global software development. J Comput Lang. 2019;51:112-130. https://doi.org/10.1016/j.cola.2018.12.005

30. Khan AA, Keung J, Niazi M, Hussain S, Shameem M. GSEPIM: a roadmap for software process assessment and improvement in the domain of global software development. J Softw Evol Process. 2019;31(1). https://doi.org/10.1002/smr.1988

31. Alves C, Oliveira J, Jansen S, 'Software ecosystems governance: a systematic literature review and research agenda', 2017. https://doi.org/10.5220/ 0006269402150226.

32. Luna AJ, Kruchten P, Pedrosa ML, Neto HR, de Moura HP. State of the art of agile governance: a systematic review. IJCSIT. 2014;6(5):121-141. https://doi.org/10.5121/ijcsit.2014.6510

33. Kitchenham B, Pearl Brereton O, Budgen D, Turner M, Bailey J, Linkman S. Systematic literature reviews in software engineering-a systematic literature review. Inf Softw Technol. 2009;51(1):7-15. https://doi.org/10.1016/j.infsof.2008.09.009

34. Budgen D, Turner M, Brereton P, Kitchenham B. Using mapping studies in software engineering. Proc PPIG 2008. $2008 ; 2$.

35. Petersen K, Vakkalanka S, Kuzniarz L. Guidelines for conducting systematic mapping studies in software engineering: an update. Inf Softw Technol. 2015;64:1-18. https://doi.org/10.1016/j.infsof.2015.03.007

36. Zamboni A, Di Thommazo A, Hernandes E, Fabbri S, 'StArt uma ferramenta computacional de apoio à revisão sistemática', in Proc.: Congresso Brasileiro de Software (CBSoft'10), Salvador, Brazil, 2010

37. Wohlin C. 'Guidelines for snowballing in systematic literature studies and a replication in software engineering', in Proceedings of the 18th International Conference on Evaluation and Assessment in Software Engineering, New York, NY, USA, 2014, pp. 38:1-38:10. https://doi.org/10.1145/2601248. 2601268.

38. Ali S, Green P. Effective information technology (IT) governance mechanisms: an IT outsourcing perspective. Inf Syst Front. 2012;14(2):179-193. https://doi.org/10.1007/s10796-009-9183-y

39. Andriole SJ. Who owns IT? Commun ACM. 2015;58(3):50-57. https://doi.org/10.1145/2660765

40. Bavani R. 'Governance patterns in global software engineering: best practices and lessons learned', in 2011 IEEE Sixth International Conference on Global Software Engineering, 2011, pp. 50-54. https://doi.org/10.1109/ICGSE.2011.17.

41. Doležel M, Buchalcevová A. Test Governance Framework for contracted IS development: ethnographically informed action research. Inf Softw Technol. Sep. 2015;65:69-94. https://doi.org/10.1016/j.infsof.2015.03.003

42. Gewald H, Helbig K. 'A governance model for managing outsourcing partnerships: a view from practice', in Proceedings of the 39th Annual Hawaii International Conference on System Sciences (HICSS'06), 2006, vol. 8, pp. 194c-194c. https://doi.org/10.1109/HICSS.2006.12.

43. Johansson B. Improving ICT governance by reorganizing operation of ICT and software applications: the first step to outsource. In: Advances in Information Systems Development; 2006:927-938.

44. Magnusson C, Chou S-C. 'Risk and compliance management framework for outsourced global software development', presented at the proceedings 5th International Conference on Global Software Engineering. ICGSE. 2010;2010:228-233. https://doi.org/10.1109/ICGSE.2010.34

45. Manteli C, van den Hooff $\mathrm{B}$, van Vliet $\mathrm{H}$. The effect of governance on global software development: an empirical research in transactive memory systems. Inf Softw Technol. 2014;56(10):1309-1321. https://doi.org/10.1016/j.infsof.2014.04.012

46. Manteli C, Hooff B, Tang A, Vliet H, 'The impact of multi-site software governance on knowledge management', in 2011 IEEE Sixth International Conference on Global Software Engineering, 2011, pp. 40-49. https://doi.org/10.1109/ICGSE.2011.16.

47. Noordeloos R, Manteli C, Van Vliet H. 'From RUP to Scrum in global software development: a case study', presented at the Proceedings - 2012 IEEE 7th International Conference on Global Software Engineering. ICGSE. 2012;2012:31-40. https://doi.org/10.1109/ICGSE.2012.11

48. Ramasubbu N, Balan RK. 'Towards governance schemes for distributed software development projects', in Proceedings of the 1st International Workshop on Software Development Governance, New York, NY, USA, 2008, pp. 11-14. https://doi.org/10.1145/1370720.1370725.

49. Šablis A, Šmite D. 'Agile teams in large-scale distributed context-isolated or connected?', presented at the ACM International Conference Proceeding Series, 2016, vol. 24-May-2016. https://doi.org/10.1145/2962695.2962705.

50. Srinivasan J, Lofgren A, Norstrom C, Lundqvist K. 'Lessons learned from a workshop on relationship building', in 2009 Fourth IEEE International Conference on Global Software Engineering, 2009, pp. 115-120. https://doi.org/10.1109/ICGSE.2009.19.

51. Vlietland J, van Vliet H. Towards a governance framework for chains of Scrum teams. Inf Softw Technol. Jan. 2015;57:52-65. https://doi.org/10.1016/ j.infsof.2014.08.008

52. Bass M, Herbsleb J, Lescher C. 'A coordination risk analysis method for multi-site projects: experience report', 2009, pp. 31-40. https://doi.org/10. 1109/ICGSE.2009.11.

53. Ebert C, Murthy BK, Jha NN. 'Managing risks in global software engineering: principles and practices', in 2008 IEEE International Conference on Global Software Engineering, 2008, pp. 131-140. https://doi.org/10.1109/ICGSE.2008.12.

54. Sinha VS, Sengupta B, Ghosal S. 'An adaptive tool integration framework to enable coordination in distributed software development', presented at the Proceedings - International Conference on Global Software Engineering. ICGSE. 2007;2007:151-155. https://doi.org/10.1109/ICGSE.2007.8 
55. Sriram R, Mathew SK. 'Global software development using agile methodologies: a review of literature', presented at the 2012 IEEE 6th International Conference on Management of Innovation and Technology. ICMIT. 2012;2012:389-393. https://doi.org/10.1109/ICMIT.2012.6225837

56. Hofstede G. Cultures and Organizations: Software of the Mind. McGraw-Hill; 1997.

57. Wohlin C, Runeson P, Höst M, Ohlsson MC, Regnell B, Wesslén A. Experimentation in Software Engineering. Berlin Heidelberg: Springer-Verlag; 2012.

58. Marinho M, Luna A, Beecham S. Global software development: practices for cultural differences. In: Lecture Notes in Computer Science (including subseries Lecture Notes in Artificial Intelligence and Lecture Notes in Bioinformatics), vol. 11271. LNCS; 2018:299-317. https://doi.org/10. 1007/978-3-030-03673-7_22.

59. Silveira KK, Prikladnicki R. 'A systematic mapping study of diversity in software engineering: a perspective from the agile methodologies', in Proceedings of the 12th International Workshop on Cooperative and Human Aspects of Software Engineering, Piscataway, NJ, USA, 2019, pp. 7-10. https://doi. org/10.1109/CHASE.2019.00010.

How to cite this article: Manjavacas A, Vizcaíno A, Ruiz F, Piattini M. Global software development governance: Challenges and solutions. J Softw Evol Proc. 2020;e2266. https://doi.org/10.1002/smr.2266

\section{APPENDIX A.: $0.1 \quad$ Control Objectives for Information and Related Technology governance objectives}

The governance objectives set out in COBIT $2019^{20}$ are listed in its Chapter 4. These objectives are grouped within the evaluate, direct, and monitor (EDM) domain, whose name expresses the main purpose and areas of activity of the objectives the domain contains. These objectives are summarized in Table A1. EDM objectives description.

TABLE A1 EDM objectives description

\begin{tabular}{cc} 
& Objective \\
COBIT 2019 & EDM01: Ensure \\
EDM- & Governance \\
Evaluate, & Framework Setting \\
direct, and & and Maintenance \\
monitor & \\
\hline
\end{tabular}

\begin{abstract}
Description
Analyze and articulate the requirements for the governance of enterprise information and technology (I\&T). Put in place and maintain governance components with clarity of authority and responsibilities to achieve the enterprise's mission, goals, and objectives.
\end{abstract}

\author{
EDM02: Ensure \\ Benefits Delivery \\ EDM03: Ensure Risk \\ Optimization
}

EDM04: Ensure Resource Optimization

EDM05: Ensure Stakeholder Engagement
Optimize the value to the business from investments in business processes, I\&T services, and I\&T assets.

Ensure that the enterprise's risk appetite and tolerance are understood, articulated, and communicated and that risk to enterprise value related to the use of I\&T is identified and managed.

Ensure that adequate and sufficient business and I\&T-related resources (people, process, and technology) are available to support enterprise objectives effectively and at optimal cost.

Ensure that stakeholders are identified and engaged in the I\&T governance system and that enterprise I\&T performance and conformance measurement and reporting are transparent, with stakeholders approving the goals and metrics and necessary remedial actions.

\section{Purpose}

Provide a consistent approach integrated and aligned with the enterprise governance approach. I\&T-related decisions are made in line with the enterprise's strategies and objectives and desired value is realized. To that end, ensure that I\&T-related processes are overseen effectively and transparently; compliance with legal, contractual, and regulatory requirements is confirmed; and the governance requirements for board members are met.

Secure optimal value from I\&T-enabled initiatives, services, and assets; cost-efficient delivery of solutions and services; and a reliable and accurate picture of costs and likely benefits so that business needs are supported effectively and efficiently.

Ensure that I\&T-related enterprise risk does not exceed the enterprise's risk appetite and risk tolerance; the impact of I\&T risk to enterprise value is identified and managed, and the potential for compliance failures is minimized.

Ensure that the resource needs of the enterprise are met in the optimal manner, I\&T costs are optimized, and there is an increased likelihood of benefit realization and readiness for future change.

Ensure that stakeholders are supportive of the I\&T strategy and road map, communication to stakeholders is effective and timely, and the basis for reporting is established to increase performance. Identify areas for improvement and confirm that I\&T-related objectives and strategies are in line with the enterprise's strategy. 


\section{APPENDIX B.: $0.1 \quad$ Search strings}

\section{- Scopus/IEEE}

TITLE-ABS-KEY ("governance" AND ("global software development" OR "distributed software development" OR "global software engineering" OR "gSd" OR "gse" OR "distributed software development" OR "distributed software engineering" OR "multi-site software development" OR "multi-site software engineering") AND "software") AND (LIMIT-TO (PUBSTAGE, "final")) AND (LIMIT-TO (SUBJAREA, "COMP")) AND (LIMIT-TO (LANGUAGE, "English"))

- $\mathrm{ACM}$

+("governance") + ("global software development" "distributed software development" "global software engineering" "gsd" "gse" "multi-site software development" "multi-sitesoftware engineering" "distributed software development" "distributed software engineering") + ("software")

\section{APPENDIX C.: $0.1 \quad$ Mapping results}

EDM01: Ensure Governance Framework Setting and Maintenance.

EDM02: Ensure Benefits Delivery.

EDM03: Ensure Risk Optimization.

EDM04: Ensure Resource Optimization.

EDM05: Ensure Stakeholder Engagement.

PS: Processes

OS: Organizational structures

TAB LE C1 Control Objectives for Information and Related Technology (COBIT) objectives mapping

\begin{tabular}{|c|c|c|c|c|c|}
\hline & \multicolumn{5}{|c|}{ COBIT objectives } \\
\hline 17 & $x$ & & $x$ & & $x$ \\
\hline 47 & $x$ & $x$ & & $x$ & $x$ \\
\hline 55 & & & & & $x$ \\
\hline 44 & $x$ & $x$ & $x$ & $x$ & $x$ \\
\hline 54 & & & & $x$ & \\
\hline 48 & $x$ & & & & \\
\hline 59 & $x$ & & & & $x$ \\
\hline 14 & $x$ & & & & $x$ \\
\hline 46 & $x$ & & & $x$ & $x$ \\
\hline 50 & $x$ & $x$ & & $x$ & $x$ \\
\hline 51 & $x$ & & & & $x$ \\
\hline 41 & $x$ & & & $x$ & $x$ \\
\hline 43 & $x$ & $x$ & & & $x$ \\
\hline 42 & $x$ & $x$ & $x$ & & $x$ \\
\hline 39 & $x$ & & & & $x$ \\
\hline
\end{tabular}


TA B LE C2 Control Objectives for Information and Related Technology (COBIT) components mapping

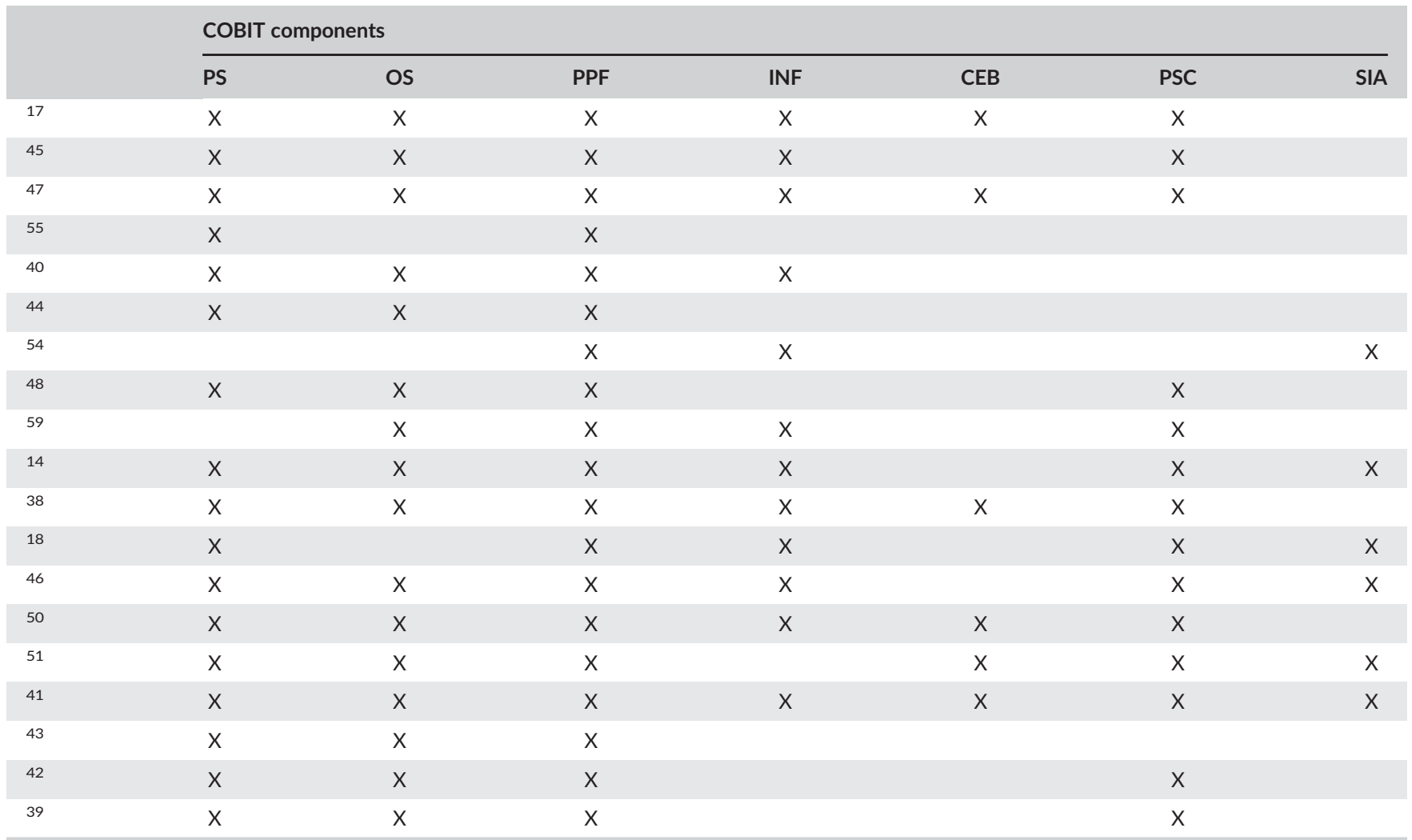

TAB LE C 3 Percentage of papers for each component and objective

\begin{tabular}{|lccccccc|} 
& PS & OS & PPF & INF & CEB & PSC \\
\hline EDM01 & 64.71 & 88.24 & 100 & 64.71 & 35.29 & 82.35 & 29.41 \\
\hline EDM02 & 83.33 & 83.33 & 100 & 66.67 & 50 & 66.67 & 16.67 \\
\hline EDM03 & 100 & 100 & 100 & 33.33 & 33.33 & 66.67 & 0 \\
\hline EDM04 & 85.71 & 85.71 & 100 & 85.71 & 57.14 & 71.43 & 42.86 \\
\hline EDM05 & 93.75 & 87.5 & 100 & 62.5 & 31.25 & 75 \\
\hline
\end{tabular}

PPF: Principles, policies, and frameworks

INF: Information

CEB: Culture, ethics, and behavior

PSC: People, skills, and competencies

SIA: Services, infrastructure, and applications

\section{APPENDIX D.: 0.1 | Quality criteria assurance}

Table TD1 shows the publications obtained through the search string, together with those gathered through snowballing. Each of the columns lists the fulfilled quality criteria finally agreed upon by consensus of the authors $(Y=$ Yes $(+1) ; P=P a r t l y(+0.5) ; N=N o(+0))$

[MP1] Bass, Matthew, James Herbsleb, and Christian Lescher. 'A coordination risk analysis method for multi-site projects: experience report', 31-40, 2009. https://doi.org/10.1109/ICGSE.2009.11.

[MP2] Šablis, A., and D. Šmite. 'Agile teams in large-scale distributed context-isolated or connected?', Vol. 24-May-2016, 2016. https://doi.org/ $10.1145 / 2962695.2962705$. 
[MP3] Noll, J., S. Beecham, I. Richardson, and C.N. Canna. 'A global teaming model for global software development governance: a case study', 179-88, 2016. https://doi.org/10.1109/ICGSE.2016.33.

[MP4] Gewald, H., and K. Helbig. 'A governance model for managing outsourcing partnerships: a view from practice'. In Proceedings of the 39th Annual Hawaii International Conference on System Sciences (HICSS'06), 8:194c-194c, 2006. https://doi.org/10.1109/HICSS.2006. 12.

[MP5] Sinha, V.S., B. Sengupta, and S. Ghosal. 'An adaptive tool integration framework to enable coordination in distributed software development', 151-55, 2007. https://doi.org/10.1109/ICGSE.2007.8.

[MP6] Bush, Ashley A., Amrit Tiwana, and Hiroshi Tsuji. 'An empirical investigation of the drivers of software outsourcing decisions in japanese organizations'. Information and Software Technology 50, no. 6 (1 May 2008): 499-510. https://doi.org/10.1016/j.infsof.2007.08.006.

[MP7] Chauhan, Muhammad Aufeef. 'A reference architecture for providing tools as a service to support global software development'. In Proceedings of the WICSA 2014 Companion Volume, 16:1-16:6. WICSA'14 Companion. New York, NY, USA: ACM, 2014. https://doi.org/10. $1145 / 2578128.2588485$

[MP8] Singi, Kapil, Pradeepkumar D S, Vikrant Kaulgud, and Sanjay Podder. 'Compliance adherence in distributed software delivery: a blockchain approach'. In Proceedings of the 13th International Conference on Global Software Engineering, 131-132. ICGSE'18. New York, NY, USA: ACM, 2018. https://doi.org/10.1145/3196369.3196383.

[MP9] Licorish, S.A., and S.G. Macdonell. 'What can developers' messages tell us? A psycholinguistic analysis of jazz teams' attitudes and behavior patterns', 107-16, 2013. https://doi.org/10.1109/ASWEC.2013.22.

[MP10] Tamburri, Damian A., Remco De Boer, Elisabetta Di Nitto, Patricia Lago, and Hans van Vliet. 'Dynamic networked organizations for software engineering'. In Proceedings of the 2013 International Workshop on Social Software Engineering, 5-12. SSE 2013. New York, NY, USA: ACM, 2013. https://doi.org/10.1145/2501535.2501537.

[MP11] Ali, Syaiful, and Peter Green. 'Effective information technology (IT) governance mechanisms: an IT outsourcing perspective'. Information Systems Frontiers 14, no. 2 (1 April 2012): 179-93. https://doi.org/10.1007/s10796-009-9183-y.

[MP12] Cataldo, Marcelo, and James D. Herbsleb. 'End-to-end features as meta-entities for enabling coordination in geographically distributed software development'. In Proceedings of the 2009 ICSE Workshop on Software Development Governance, 21-26. SDG'09. Washington, DC, USA: IEEE Computer Society, 2009. https://doi.org/10.1109/SDG.2009.5071332.

[MP13] Femmer, H., M. Kuhrmann, J. Stimmer, and J. Junge. 'Experiences from the design of an artifact model for distributed agile project management', 1-5, 2014. https://doi.org/10.1109/ICGSE.2014.9.

[MP14] Noordeloos, R., C. Manteli, and H. Van Vliet. 'From RUP to Scrum in global software development: a case study', 31-40, 2012. https:// doi.org/10.1109/ICGSE.2012.11.

[MP15] Sriram, R., and S.K. Mathew. 'Global software development using agile methodologies: a review of literature', 389-93, 2012. https://doi. org/10.1109/ICMIT.2012.6225837.

[MP16] Dubinsky, Y., S. Ravid, A. rafaeli, and R. Bar-Nahor. 'Governance mechanisms in global development environments'. In 2011 IEEE Sixth International Conference on Global Software Engineering, 6-14, 2011. https://doi.org/10.1109/ICGSE.2011.28.

[MP17] Bavani, R. 'Governance patterns in global software engineering: best practices and lessons learned'. In 2011 IEEE Sixth International Conference on Global Software Engineering, 50-54, 2011. https://doi.org/10.1109/ICGSE.2011.17.

[MP18] Johansson, Björn. 'Improving ICT governance by reorganizing operation of ICT and software applications: the first step to outsource'. In Advances in Information Systems Development, edited by Anders G. Nilsson, Remigijus Gustas, Wita Wojtkowski, W. Gregory Wojtkowski, Stanisław Wrycza, and Jože Zupančič, 927-38. Springer US, 2006.

[MP19] Srinivasan, J., A. Lofgren, C. Norstrom, and K. Lundqvist. 'Lessons learned from a workshop on relationship building'. In 2009 Fourth IEEE International Conference on Global Software Engineering, 115-20, 2009. https://doi.org/10.1109/ICGSE.2009.19.

[MP20] Singh, R., and K. Hofmann. 'Managing global R \& D projects: practical experience in building project management competency', 185-89, 2012. https://doi.org/10.1109/ICGSE.2012.23.

[MP21] Magnusson, C., and S.-C. Chou. 'Risk and compliance management framework for outsourced global software development', 228-33, 2010. https://doi.org/10.1109/ICGSE.2010.34

[MP22] Bannerman, P. L. 'Software development governance: a meta-management perspective'. In 2009 ICSE Workshop on Software Development Governance, 3-8, 2009. https://doi.org/10.1109/SDG.2009.5071329.

[MP23] Doležel, Michal, and Alena Buchalcevová. 'Test governance framework for contracted IS development: ethnographically informed action research'. Information and Software Technology 65 (1 September 2015): 69-94. https://doi.org/10.1016/j.infsof.2015.03. 003.

[MP24] Manteli, C., B. Van Den Hooff, and H. Van Vliet. 'The effect of governance on global software development: an empirical research in transactive memory systems'. Information and Software Technology 56, no. 10 (2014): 1309-21. https://doi.org/10.1016/j.infsof.2014.04. 012. 
[MP25] Manteli, C., B. v d Hooff, A. Tang, and H. v Vliet. 'The impact of multi-site software governance on knowledge management'. In 2011 IEEE Sixth International Conference on Global Software Engineering, 40-49, 2011. https://doi.org/10.1109/ICGSE.2011.16.

[MP26] Vlietland, Jan, and Hans van Vliet. 'Towards a governance framework for chains of Scrum teams'. Information and Software Technology 57 (1 January 2015): 52-65. https://doi.org/10.1016/j.infsof.2014.08.008.

[MP27] Ramasubbu, Narayan, and Rajesh Krishna Balan. 'Towards governance schemes for distributed software development projects'. In Proceedings of the 1st International Workshop on Software Development Governance, 11-14. SDG'08. New York, NY, USA: ACM, 2008. https://doi.org/10.1145/1370720.1370725.

[MP28] Andriole, Stephen J. 'Who owns IT?' Commun. ACM 58, no. 3 (February 2015): 50-57. https://doi.org/10.1145/2660765.

TABLE D1 Quality assessments

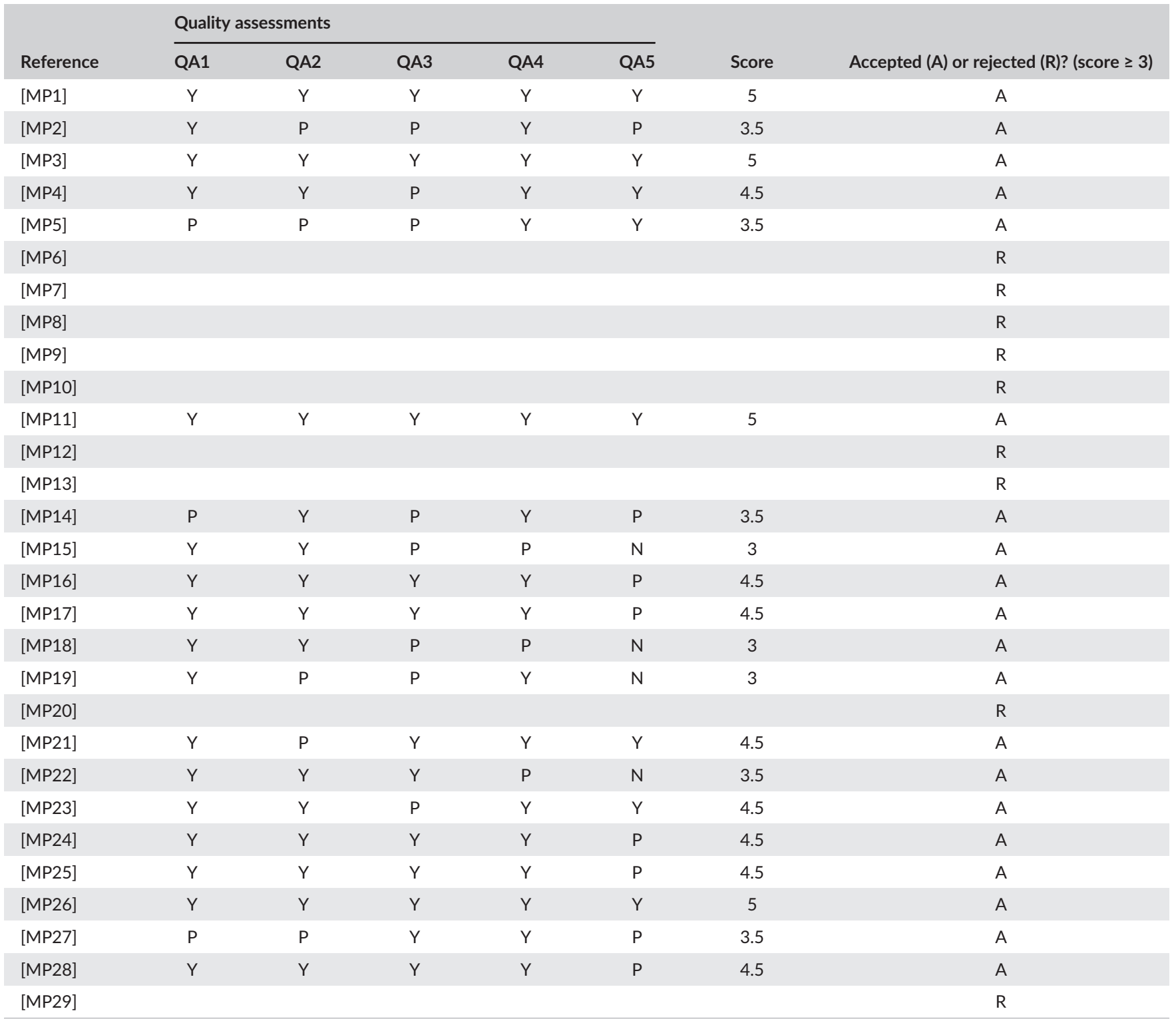

[MP29] Licorish, S.A., and S.G. Macdonell. 'Differences in jazz project leaders' competencies and behaviors: a preliminary empirical investigation', 1-8, 2013. https://doi.org/10.1109/CHASE.2013.6614725. 